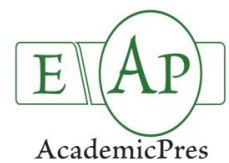

\title{
Evaluation of Drought Tolerance Indices for Selection of Confectionery Sunflower (Helianthus anuus L.) Landraces under Various Environmental Conditions
}

\author{
Esmaeil GHOLINEZHAD ${ }^{1 *}$, Reza DARVISHZADEH ${ }^{2}$, Iraj BERNOUSI ${ }^{2}$ \\ ${ }^{1}$ Department of Agronomy, Payame Noor University, Tehran, Iran; Gholinezhad1358@yahoo.com (*corresponding author) \\ ${ }^{2}$ Department of Plant Breeding and Biotechnology,Urmia University,Urmia, Iran; r.darvishzadeh@urmia.ac.ir,ibernousi@gmail.com
}

\begin{abstract}
In order to study the response of 56 landraces of confectionery sunflower to drought stress, an experiment was conducted in the experimental field of Agricultural Research Center of West-Azerbaijan in 2012-2013 cropping seasons. The genotypes were evaluated in three separated rectangular $7 \times 8$ lattice design with two replications in three irrigation treatments including optimum irrigation, moderate stress and sever stress where irrigation was done after depletion of $50 \%, 70 \%$ and $90 \%$ of available water, respectively. Nine drought tolerance indices including mean productivity (MP), stress tolerance index (STI), geometric mean productivity (GMP), harmonic mean (HARM), stress non-stress production index (SNPI), yield index (YI), drought resistance index $(\mathrm{DI})$, modified stress tolerance index in optimum irrigation $\left(\mathrm{M}_{\mathrm{P}} \mathrm{STI}\right)$, and modified stress tolerance index in moderate and severe stress $\left(\mathrm{M}_{s} \mathrm{STI}\right)$ were calculated based on grain yield under well-watered $\left(\mathrm{Y}_{\mathrm{P}}\right)$, moderate drought

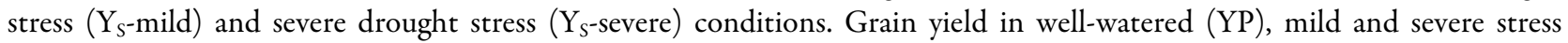
conditions was significantly and positively correlated with MP, STI, GMP, HARM, SNPI, YI, DI, $M_{P} S T I$ and $M_{S} S T I$. Results of this study showed that in moderate drought stress conditions the indices MP, STI, GMP and HARM could be used as the most suitable indicators for screening drought tolerant genotypes. Based on cluster analysis, the studied genotypes were grouped in 3 clusters in each one of water treatment conditions. To select drought tolerant genotypes in severe drought stress conditions, it is suggested to use indices MP, STI, GMP, HARM, SNPI and $M_{S} S T I$ because these indices supports stable and high yield in both non-stress and stress conditions.
\end{abstract}

Keywords: biplot presentation, confectionary sunflower, cluster analysis, landraces

\section{Introduction}

Sunflower (Helianthus annuus L.) is one of the 67 species in the genus Helianthus. Nowadays, two main types of sunflower are grown: for oilseed production and nonoilseed or confectionery types (Salunkhe et al., 1999). Confectionery sunflower produces large seeds with low oil content and is used in baking and snack applications ( $\mathrm{Lu}$ and Hoeft, 2007). Confectionery kernels are roasted and salted, or roasted and no salt added and marketed as edible chips. The confectionery type is one of the most popular and important crops in Iran. It is cultivated in all parts of the country especially in the northwest regions. Abiotic stresses such as cold, salinity, heat and water stress is the principal cause of crop failure worldwide which dipping average yields by more than 50\% (Jaleel, 2007).

The different strategies have been suggested for selecting resistant and relatively resistant genotypes to drought stress. Fisher and Maurer (1978) stated that seed yield in drought environment could be considered as drought resistant index. Blum (1988) reported that selection of genotypes for drought resistant must be associated with high yield in non- stress environments. Drought indices based on loss of yield under stress conditions compared to normal state have been used for screening drought tolerant genotypes (Mitra, 2001). Several selection indices such as geometric mean productivity (GMP) and stress tolerance index (STI) by Fernandez (1992), mean productivity (MP) and tolerance index (TOL) by Rosielle and Hamblin (1981), harmonic mean (HARM) by Jafari et al. (2009), stress susceptibility index (SSI) by Fisher and Maurer (1978), yield stability index (YSI) by Bouslama and Schapaugh (1984) and yield index (YI) by (Gavuzzi et al., 1997) were used for screening drought tolerant genotypes.

Khodarahmpour et al. (2011) showed that the STI and GMP indices were the more accurate criteria for selection of heat tolerant and high yielding genotypes. Eivazy et al. (2013) indicated that MP index was the best criterion for selecting genotypes with high grain yield at both well watered and drought stressed conditions. According to Ganjali et al. (2009), there were positive and highly significant correlations among MP, GMP, STI and HARM with yield in stressed and non-stressed conditions. Jabbari $e t$ al. (2008) studied drought tolerant indices in sunflower 
188

genotypes and found significant and positive correlation between STI and grain yield under non-stressed as well as mild and severe drought stresses conditions. Daneshian and Jonoubi (2008) reported that MP, GMP and STI are the most useful indices for evaluating tolerance to drought stress in sunflower. In some researches, YI led to ranking genotypes based on yield in stress conditions (Sio-Se Mardeh et al., 2006). Drikvand et al. (2012) exhibited that the most suitable criteria for the identification of genotypes under irrigated and rain fed conditions were GMP, MP and STI. Anwar et al. (2011) observed that there were positive and significant correlations between grain yield and MP, HARM, GMP, STI and K1STI under irrigated and stressed conditions. Farshadfar et al. (2013) reported that $\mathrm{K}_{1} S T I$, $\mathrm{K}_{2} \mathrm{STI}$, SSPI, RDI, ATI, SNPI and DI could be used as the most suitable indicators for screening drought tolerant cultivars in wheat. Pourdad et al. (2008) indicated that cluster analysis based on STI, MP, GMP, YS and YP classify genotypes into three groups with different susceptibility to drought stress.

According to Fernandez (1992) report, genotypes can be classified into four groups based on their performance in stressed and non-stressed conditions: group A: genotypes with high yield under both stressed and non-stressed conditions, group B: genotypes with high yield under nonstressed conditions, group $\mathrm{C}$ : genotypes with high yield under stressed conditions, group D: genotypes with poor performance under both stressed and non-stressed conditions. A suitable index must be able to distinguish group A from the other groups. Biplot is an exploratory data visualization technique to display the multivariate data into a two dimensional scatter plot. The concept of biplot was first developed by Gabriel (1971). This technique has extensively been used in the analysis of multi-environmental trails (Ahmadi et al., 2000; Farshadfar et al., 2001; Golabadi et al., 2006). The objectives of the present study were to evaluate several drought tolerance indices and identify drought- tolerant genotypes in confectionery sunflower.

\section{Materials and methods}

In order to evaluate the effect of drought stress on grain yield of confectionery sunflower, an experiment was carried out in the experimental field of Agricultural Research center of West-Azerbaijan in 2012-2013. 56 confectionery sunflower accessions were evaluated in three separated rectangular $7 \times 8$ lattice design with two replications under three irrigation treatments including optimum irrigation (irrigation after depletion of $50 \%$ of available water), moderate stress (irrigation after depletion of $70 \%$ of available water) and sever stress (irrigation after depletion of $90 \%$ of available water).

The soil physical and chemical characteristic of the experimental area is presented in Tab. 1. Mean monthly rainfall, humidity and temperature were recorded through the growing period (Tab. 2). Soil moisture samples were taken from 0-30 and 30-60 cm depth. Weight moisture percentage was determined by pressure plate (armfield CAT.REF: FEL13B-1 Serial Number: 6353 A 24S98). Field capacity and wilting point were 26 and 14 , respectively. In order to obtain the irrigation times, soil samples were taken by auger from root development depth in each irrigation regimes 48 hours post irrigation. Irrigation intervals were determined from soil moisture percentage 20 , 17.6 and 15.2 days for severe, moderate and well-watered conditions, respectively. Irrigation volume was calculated by equation 1 (Alizadeh, 2009):

$$
V=\frac{\left(f_{\mathcal{C}}-\theta m\right) \times \rho \times \operatorname{Droot} \times A}{E i}
$$

where:

$\mathrm{V}=$ irrigation water volume $\left(\mathrm{m}^{3}\right), \Theta \mathrm{m}=$ soil moisture weight percentage per replication, $A=$ irrigated area $\left(\mathrm{m}^{2}\right)$, $\mathrm{FC}=$ field capacity, $\rho \mathrm{m}=$ soil density $\left(\mathrm{g} \mathrm{cm}^{-3}\right)$, Droot $=$ developmental root depth (m).

Required water volume per irrigation regimes calculated and distributed equally by flume and chronometer with water distribution efficiency of 90 percent. Plants were harvested at maturity, and then the grain yield was recorded for every plot. Harvesting area was $3.6 \mathrm{~m}^{2}$ from the two middle lines. The drought tolerance indices were calculated for every genotype using the corresponding well-watered and water-stressed plots. Drought tolerance indices were calculated by using the equations cited in Tab. 3 .

Analysis of variance performed using PROC GLM in the SAS software (SAS Institute Inc., Cary, NC, USA). The comparison of the means was done by Tukey's test at 5\% probability level. Correlations between grain yield per plot in each of the water regimes and drought tolerance indices were determined using SAS PROC CORR. The classification of genotypes using grain yield per plot in each of the water regimes and drought tolerance indices data was performed by Ward algorithm based on squared Euclidean distances. Biplot analysis was used to classify the drought tolerance indices as well as the confectionery sunflower landraces (Mohammadi et al., 2010). Data processing was performed using SPSS 20.00 statistical software (SPSS/PC20, SPSS Inc., Chicago, IL, USA; http://www.spss.com).

Tab. 1. Chemical and physical properties of farm soil at depth of $0-30 \mathrm{~cm}$

\begin{tabular}{ccccccccccccc}
\hline $\begin{array}{c}\text { Soil } \\
\text { texture }\end{array}$ & $\begin{array}{c}\text { Soil } \\
\text { density } \\
\mathrm{g} \mathrm{cm}^{-3}\end{array}$ & $\begin{array}{c}\text { Electrical } \\
\text { conductivity } \\
(\mathrm{d} / \mathrm{m})\end{array}$ & $\mathrm{pH}$ & $\begin{array}{c}\text { Percentage } \\
\text { of } \\
\text { saturation } \\
(\%)\end{array}$ & $\begin{array}{c}\text { Lime } \\
(\%)\end{array}$ & $\begin{array}{c}\text { Clay } \\
(\%)\end{array}$ & $\begin{array}{c}\text { Silt } \\
(\%)\end{array}$ & $\begin{array}{c}\text { Sand } \\
(\%)\end{array}$ & $\begin{array}{c}\text { Carbon } \\
\text { organic } \\
(\%)\end{array}$ & $\begin{array}{c}\text { Nitrogen } \\
(\%)\end{array}$ & $\begin{array}{c}\text { Phosphor } \\
(\mathrm{ppm})\end{array}$ & $\begin{array}{c}\text { Potassium } \\
(\mathrm{ppm})\end{array}$ \\
\hline $\begin{array}{c}\text { Clay } \\
\text { loam }\end{array}$ & 1.4 & 0.8 & 8 & 47 & 17 & 35 & 37 & 28 & 1.2 & 0.12 & 12 & 375 \\
\hline
\end{tabular}


Tab. 2. Monthly climate data during sunflower growing season

\begin{tabular}{|c|c|c|c|c|c|c|}
\hline \multirow{2}{*}{ Meteorological Parameters } & \multicolumn{6}{|c|}{ Month } \\
\hline & Mars & April & May & June & July & August \\
\hline Maximum Temperature $\left({ }^{\circ} \mathrm{C}\right)$ & 16.6 & 23.3 & 28.2 & 30.1 & 33.1 & 28.6 \\
\hline Minimum Temperature $\left({ }^{\circ} \mathrm{C}\right)$ & 3.1 & 8.3 & 11.9 & 15.3 & 16.2 & 13.1 \\
\hline Mean Temperature $\left({ }^{\circ} \mathrm{C}\right)$ & 9.9 & 15.8 & 20 & 22.7 & 24.6 & 20.9 \\
\hline Total Rainfall (mm) & 31.9 & 15 & 18.8 & 9.2 & 1.8 & 8.4 \\
\hline Total Evaporation (mm) & 81.9 & 181.9 & 255.9 & 269.3 & 263.4 & 200.4 \\
\hline Mean Relative Humidity (\%) & 58 & 56 & 48 & 52 & 46 & 52 \\
\hline
\end{tabular}

Tab. 3. Names of local landraces

\begin{tabular}{clclll}
\hline & & & \multicolumn{2}{c}{ Names of local landraces } \\
\hline No. & & No. & & No. & \\
\hline 1 & Saghez 1 & 20 & Salmas 2 & 39 & Hamadan 2 \\
2 & Anghane 4 & 21 & Vaghaslou-Olya 4 & 40 & Shabestar-Kouzeh-Kanan 3 \\
3 & Urmia-Barouj & 22 & Salmas-Gharaghashlagh-Pesteii & 41 & Saghez 4 \\
\hline 4 & Urmia-Maranghalou & 23 & Lalalou-Torab 2 & 42 & Saghez 5 \\
\hline 5 & Marand-Dizaj- Ghalami & 24 & Shirabad 2 & 43 & Saghez 3 \\
6 & Jabalkandi 2 & 25 & Gharagoz 1 & 44 & Shahroud 2 \\
7 & Salmas - Sadaghian & 26 & Vaghaslou-Sofla 1 & 45 & Alibaglou 1 \\
8 & Babaghanje 6 & 27 & Khanneshan 1 & 46 & Baneh 2 \\
\hline 9 & Miyaneh-Basin & 28 & Heydarlou 1 & 47 & Salmas-Gharagheshlagh- Ghalami \\
10 & Boucan & 29 & Saribaglou 5 & 48 & Marand-1389-2 \\
11 & Urmia - Nuoshinshahr & 30 & Chongharalou-Yekan 4 & 49 & Salmas-Gharaghashlagh- Badami \\
12 & Karimabad & 31 & Maranghalou 6 & 50 & Shabestar-Kouzeh Kanan 1 \\
13 & Vaghaslou-Olya 1 & 32 & Abajalou 1 & 51 & Sanandaj \\
14 & Vaghaslou-Olya 3 & 33 & Hamadan 1 & 52 & Shabestar-Kouzeh-Kanan 2 \\
15 & Ordoshahi 1 & 34 & Saghez 2 & 53 & Baneh 3 \\
16 & Marana-Yamchi-Pesteii & 35 & Piranshahr-Serokani & 54 & Piranshahr-Baleban \\
\hline 17 & Mazandaran-Tirtash & 36 & Piranshahr Andizeh & 55 & Baneh 1 \\
\hline 18 & Sardasht & 37 & Mashhad & 56 & Marand-1389-1 \\
19 & Marana-Yamchi 4 & 38 & Shahroud 1 & & \\
\hline
\end{tabular}

\section{Results and discussion}

\section{Comparing landraces based on the resistance / tolerance indices}

To investigate suitable drought tolerance indices for screening confectionery sunflower landraces under drought stress conditions, different indices were calculated based on grain yield under stressed and non-stressed environments (Tab. 6 and 7). A suitable index must have a significant correlation with grain yield under both non-stress and stress conditions (Mitra, 2001). Based on the stress tolerance index (STI) and grain yield, the landraces: 2, 12, 14, 16, 25, $28,29,31,32,33,47,49$ and 56 were found drought tolerance with the highest STI and grain yield under mild stressed and non-stressed conditions while the landraces: 37 and 38 displayed the lowest amount of STI and grain yield under mild stressed and non-stressed conditions (Tab. 6). Landraces: 2, 7 and 12 had the most STI and grain yield under severe and non-stress conditions while the landraces 36 and 37 had the lowest amount of STI and grain yield under severe and non-stress conditions (Tab. 7). Khodarahmpour et al. (2011) and Khalili et al. (2012) reported that STI and GMP indices were the more accurate criteria for selection of heat tolerant and high yielding genotypes.

The highest GMP and MP were shown in landraces: 2, 12,
$14,25,26,28,31,32,33,49$ and 56 under mild stressed and non-stressed conditions (Tab. 6). The lowest GMP and MP were observed in landraces: 36 and 37 under severe stressed and non-stressed conditions (Tab. 6). Under severe and nonstress conditions, the highest and lowest GMP and MP were observed in the accessions (2 and 12) and (36 and 37), respectively (Tab. 7). Based on Rosiele and Hamblin (1981) decline, the stress tolerance index (STI) and mean productivity (MP) are defined as the difference in yield and the average yield between stressed and non-stressed conditions, respectively.

The highest and lowest HARM were observed in the landraces: $(12,25,26,32,33,49$ and 56) and (9, 10 and $41)$, respectively, in the mild stressed and non-stressed conditions ( $\mathrm{Tab} .6)$. Under severe stressed and nonstressed conditions, the highest and lowest HARM were related to landraces: $(5,7,12$ and 29$)$ and $(36,37,40$ and 41), respectively (Tab. 7). Mevlut and Sait (2011) indicated that the genotypes with high STI usually present high difference in yield between two different conditions. They reported in general, common ranks for studied landraces by GMP, MP and STI, which suggests that these three indices are similar for screening drought tolerant landraces.

Based on the yield index (YI), the landraces 12, 25, 32 and 56 were the most tolerant landraces under mild stressed and non-stressed conditions (Tab. 6). Landraces: 5, 7, 12, 29, 31 and 43 had the highest YI under severe stressed 
190

Tab. 4. Drought tolerance indices

\begin{tabular}{|c|c|c|c|}
\hline Index name & Outcome & Equation & Reference \\
\hline Mean Productivity (MP) & $\begin{array}{c}\text { the landraces with high } \\
\text { value of this index will be } \\
\text { more desirable }\end{array}$ & $M P=\frac{Y_{S}+Y_{P}}{2}$ & (Rosielle and Hamblin, 1981) \\
\hline $\begin{array}{l}\text { Geometric Mean Productivity } \\
\text { (GMP) }\end{array}$ & $\begin{array}{l}\text { the landraces with high } \\
\text { value of this index will be } \\
\text { more desirable }\end{array}$ & $G M P=\sqrt{\left(Y_{S}\right)\left(Y_{P}\right)}$ & (Kristin et al., 1997) \\
\hline Stress Tolerance Index (STI) & $\begin{array}{l}\text { The landraces with high } \\
\text { STI values will be tolerant } \\
\text { to drought stress }\end{array}$ & $S T I=\frac{\left(Y_{S}\right)\left(Y_{P}\right)}{\left(\bar{Y}_{P}\right)^{2}}$ & (Fernandez, 1992) \\
\hline Harmonic Mean (HARM) & $\begin{array}{l}\text { the landraces with high } \\
\text { value of this index will be } \\
\text { more desirable }\end{array}$ & $H A R M=\frac{2(Y p \times Y s)}{Y p+Y s}$ & (Jafari et al., 2009) \\
\hline Yield Index (YI) & $\begin{array}{l}\text { the landraces with high } \\
\text { value of this index will be } \\
\text { suitable for drought stress } \\
\text { condition }\end{array}$ & $Y I=\frac{Y_{S}}{\overline{Y_{P}}}$ & (Gavuzzi et al., 1997) \\
\hline Drought Resistance Index (DI) & $\begin{array}{l}\text { the landraces with high } \\
\text { value of this index will be } \\
\text { suitable for drought stress } \\
\text { condition }\end{array}$ & $D I=\frac{\left[Y_{S} \times\left(\frac{Y_{S}}{Y_{P}}\right)\right]}{\overline{Y_{S}}}$ & (Lan, 1998) \\
\hline $\begin{array}{l}\text { Stress Non-Stress Production } \\
\text { Index }\end{array}$ & $\begin{array}{l}\text { the landraces with high } \\
\text { value of this index will be } \\
\text { suitable for drought stress } \\
\text { condition }\end{array}$ & $S N P I=\left[\frac{Y p+Y s}{Y_{p}-Y_{S}}\right]^{\frac{1}{3}} \times\left[Y p \times Y S \times Y Y_{s}\right]^{\frac{1}{3}}$ & (Moosavi et al., 2008) \\
\hline $\begin{array}{l}\text { Modified Stress Tolerance } \\
\text { Index in Optimum Irrigation } \\
(\mathrm{MPSTI})\end{array}$ & $\begin{array}{l}\text { the landraces with high } \\
\text { value of this index will be } \\
\text { suitable for drought stress } \\
\text { condition }\end{array}$ & $M p S T I=\frac{[(Y)] p)^{2}}{[(\bar{Y})] p)^{2}} \times S T I$ & (Farshadfar and Sutka, 2002) \\
\hline $\begin{array}{l}\text { Modified Stress Tolerance } \\
\text { Index in Moderate and Severe } \\
\text { Stress (MsSTI) }\end{array}$ & $\begin{array}{l}\text { the landraces with high } \\
\text { value of this index will be } \\
\text { suitable for drought stress } \\
\text { condition }\end{array}$ & $M s S T I=\frac{\left.[(Y)]_{s}\right)^{2}}{\left.[(\bar{Y})]_{s}\right)^{2}} \times S T I$ & (Farshadfar and Sutka, 2002) \\
\hline
\end{tabular}

$Y S$ and $Y P$ are stress and optimal (potential) yield of a given genotype, respectively. $\bar{Y}$ s and $\bar{Y}$ p are average yield of all genotypes under stress and optimal conditions, respectively.

Tab. 5. Simple and combined analyses of variance for grain yield in 56 Iranian confectionery sunflower landraces under non-stressed, moderate and severe drought stressed conditions.

\begin{tabular}{|c|c|c|c|c|c|c|}
\hline \multirow{2}{*}{ Source of variation } & \multicolumn{4}{|c|}{ Individual } & \multicolumn{2}{|c|}{ Combined } \\
\hline & $\mathrm{df}$ & MS Optimum conditions & MS $S_{\text {Mild stressed conditions }}$ & MS ${ }_{\text {Severe stressed conditions }}$ & $\mathrm{df}$ & MS \\
\hline (Environment) & - & - & - & - & 2 & $46277099.58^{* *}$ \\
\hline Replication & 1 & $1293418.65^{\circ}$ & $707.52^{\mathrm{ns}}$ & $326538^{* *}$ & - & - \\
\hline $\begin{array}{c}\text { Replication } \\
\text { (Environment) }\end{array}$ & - & - & - & - & 3 & 540221.39 \\
\hline Block (Replication) & 14 & $195166.60^{\mathrm{ns}}$ & $250894.24^{\mathrm{ns}}$ & $119807.47^{*}$ & - & - \\
\hline $\begin{array}{l}\text { Block (Environment } \times \\
\text { Replication) }\end{array}$ & - & - & - & - & 42 & $507269.85^{\circ \prime}$ \\
\hline Genotype & 55 & $878661.58 *$ & $813047.52^{*}$ & $286444.80^{*}$ & 55 & $1380455.49^{* *}$ \\
\hline $\begin{array}{c}\text { (Environment } \times \\
\text { Genotype) }\end{array}$ & - & - & - & - & 110 & $298849.20^{*}$ \\
\hline Error & 41 & 326456.17 & 263024.93 & 51214.32 & 123 & 213565.1 \\
\hline C.V.(\%) & - & 21.32 & 24.84 & 16.23 & - & 22.58 \\
\hline
\end{tabular}

${ }^{* *},{ }^{*}$ and Ns significant at the $1 \%, 5 \%$ probability levels and non significant respectively

conditions (Tab. 7). Based on $\mathrm{M}_{\mathrm{P}} \mathrm{STI}$ and $\mathrm{M}_{\mathrm{S}} \mathrm{STI}$ the landraces $2,8,12,14,25,26,32,33$ and 47 were the most tolerant under mild stressed conditions (Tab. 6). Under severe stressed conditions, the highest $\mathrm{M}_{\mathrm{P} S T I}$ and $\mathrm{M}_{\mathrm{S}} \mathrm{STI}$ were related to landraces 7, 12, 25, and 49 (Tab. 7). With regard to stress non-stress production index (SNPI) under mild and severe stressed conditions: landraces $(2,8,14,26$ and 47$)$ and $(5,7,22,29,31,34$ and 43) were the most tolerant accessions, respectively (Tab. 6 and 7). Based on drought index (DI) under mild stressed conditions, the high tolerant landraces were 12, 16, 25, 31, 32 and 56 (Tab. 6). Based on drought index (DI) under severe stressed conditions, the most tolerant landraces were 5, 7,9 and 43 (Tab. 7). Ilker et al. (2011) concluded that MP, GMP and STI are convenient indices to choose high yielding wheat genotypes in both stressed and non-stressed conditions. 
Farshadfar et al. (2013) reported that K1STI, K2STI, SNPI and DI indices can be used as the most suitable indicators for screening drought tolerant cultivars in wheat.

Totally, based on MP, STI, GMP, HARM, YI, DI and MSSTI indices and ranking method the landraces: 2, 12, 25, $26,31,32,33$ and 56 were as the most drought tolerant and the landraces: $9,10,19,35,36,37,38,40$ and 41 were as the most susceptible under mild stressed conditions (Tab. 6). Under severe stressed conditions, based on MP, STI, GMP, HARM, YI, DI, SNPI and MSSTI indices and ranking method the landraces: 2, 5, 7, 12, 29 and 31 were as the most drought tolerant and the landraces: 9, 10, 17, 35, 36, 37, 40, 41 and 54 were as the most susceptible landraces (Tab. 7).

\section{Cluster analysis}

To determine the most desirable drought tolerance criteria, the correlation coefficient between $Y_{P}, Y_{S}$ and other quantitative drought tolerance indices in mild and severe stressed conditions were calculated (Tab. 8 and 9). The results indicated that in moderate and severe drought stressed conditions there were positive and significant correlations among $Y_{P}$ and MP, STI, GMP, HARM, SNPI, YI, DI, MPSTI and MSSTI. Also in moderate and severe drought stressed conditions, there were positive and significant correlations among Ys and MP, STI, GMP, HARM, YI, DI, MPSTI and MSSTI. However, indices

Tab. 6. Resistance/tolerance indices for 56 Iranian confectionery sunflower landraces under moderate drought stressed and non-stressed conditions.

\begin{tabular}{|c|c|c|c|c|c|c|c|c|c|c|c|c|c|}
\hline No & Genotype & Rank & HARM & Rank & GMP & Rank & STI & Rank & MP & Rank & Ys-mild & Rank & Yp \\
\hline 1 & Saghez 1 & 19 & 2.60 & 19.5 & 2.65 & 19.5 & 0.93 & 18.5 & 2.71 & 22 & 2169.72 & 15 & 3241.94 \\
\hline 2 & Anghane 4 & 13 & 2.81 & 4 & 3.38 & 4 & 1.51 & 1 & 4.06 & 38 & 1807.79 & 1 & 6310.77 \\
\hline 3 & Urmia-Barouj & 25 & 2.41 & 24.5 & 2.51 & 24 & 0.84 & 23 & 2.62 & 35 & 1883.44 & 12 & 3355.65 \\
\hline 4 & Urmia-Maranghalou & 39.5 & 2.10 & 38 & 2.20 & 38 & 0.64 & 34.5 & 2.31 & 42 & 1615.43 & 26 & 3001.82 \\
\hline 5 & Marand-Dizaj-Ghalami & 27 & 2.36 & 27 & 2.42 & 27 & 0.78 & 28 & 2.49 & 33 & 1917.77 & 21 & 3055.23 \\
\hline 6 & Jabalkandi 2 & 29 & 2.35 & 31 & 2.37 & 31 & 0.74 & 31 & 2.39 & 23 & 2085.56 & 33 & 2695.38 \\
\hline 7 & Salmas - Sadaghian & 18 & 2.61 & 19.5 & 2.65 & 19.5 & 0.93 & 21 & 2.69 & 19 & 2211.75 & 17 & 3177.21 \\
\hline 8 & Babaghanje 6 & 20 & 2.59 & 17.5 & 2.68 & 17.5 & 0.95 & 15 & 2.78 & 24 & 2048.18 & 7 & 3505.74 \\
\hline 9 & Miyaneh-Basin & 54 & 1.25 & 54 & 1.25 & 54 & 0.21 & 54 & 1.25 & 52 & 1273.47 & 55 & 1221.97 \\
\hline 10 & Boucan & 52 & 1.33 & 51 & 1.43 & 51 & 0.27 & 48.5 & 1.54 & 54 & 973.88 & 43 & 2103.75 \\
\hline 11 & $\begin{array}{c}\text { Urmia - } \\
\text { Nuoshinshahr }\end{array}$ & 14.5 & 2.72 & 16 & 2.73 & 15.5 & 0.99 & 16.5 & 2.75 & 13 & 2482.86 & 25 & 3012.03 \\
\hline 12 & Karimabad & 1 & 3.67 & 1 & 3.67 & 1 & 1.78 & 2 & 3.67 & 2 & 3611.45 & 3 & 3728.20 \\
\hline 13 & Vaghaslou-Olya 1 & 42 & 1.80 & 42 & 1.92 & 42 & 0.49 & 42 & 2.05 & 47 & 1328.74 & 31 & 2768.42 \\
\hline 14 & Vaghaslou-Olya 3 & 9 & 3.04 & 9 & 3.07 & 9 & 1.25 & 9 & 3.09 & 12 & 2686.43 & 9 & 3500.27 \\
\hline 15 & Ordoshahi 1 & 23 & 2.49 & 24.5 & 2.51 & 25 & 0.83 & 25.5 & 2.53 & 20 & 2208.24 & 29 & 2852.48 \\
\hline 16 & $\begin{array}{l}\text { Marana-Yamchi- } \\
\text { Pesteii }\end{array}$ & 12 & 2.85 & 13 & 2.85 & 13.5 & 1.07 & 14 & 2.85 & 10 & 2889.97 & 30 & 2802.96 \\
\hline 17 & Mazandaran-Tirtash & 43 & 1.75 & 43 & 1.75 & 43 & 0.41 & 43 & 1.75 & 36 & 1832.44 & 50 & 1675.26 \\
\hline 18 & Sardasht & 41 & 2.05 & 41 & 2.07 & 41 & 0.57 & 41 & 2.09 & 37 & 1816.67 & 40 & 2356.11 \\
\hline 19 & Marana-Yamchi 4 & 48 & 1.50 & 48 & 1.52 & 48 & 0.31 & 48.5 & 1.54 & 50 & 1299.26 & 47 & 1783.27 \\
\hline 20 & Salmas 2 & 17 & 2.66 & 17.5 & 2.68 & 17.5 & 0.95 & 20 & 2.70 & 16 & 2367.56 & 22.5 & 3037.67 \\
\hline 21 & Vaghaslou-Olya 4 & 31 & 2.33 & 29.5 & 2.38 & 29.5 & 0.75 & 29 & 2.43 & 32 & 1927.74 & 28 & 2935.50 \\
\hline 22 & $\begin{array}{c}\text { Salmas- } \\
\text { Gharaghashlagh- } \\
\text { Pesteii }\end{array}$ & 22 & 2.52 & 23 & 2.53 & 23 & 0.85 & 25.5 & 2.53 & 15 & 2371.90 & 34 & 2691.73 \\
\hline 23 & Lalalou-Torab 2 & 21 & 2.53 & 21 & 2.62 & 21 & 0.91 & 18.5 & 2.71 & 28 & 2002.13 & 10 & 3418.40 \\
\hline 24 & Shirabad 2 & 35 & 2.27 & 33.5 & 2.29 & 33.5 & 0.70 & 34.5 & 2.31 & 26 & 2026.19 & 36 & 2591.75 \\
\hline 25 & Gharagoz 1 & 2 & 3.65 & 2 & 3.65 & 2 & 1.77 & 3 & 3.65 & 1 & 3778.83 & 5 & 3528.58 \\
\hline 26 & Vaghaslou-Sofla 1 & 4.5 & 3.27 & 5 & 3.28 & 5 & 1.43 & 5 & 3.30 & 7 & 2965.42 & 4 & 3635.39 \\
\hline 27 & Khanneshan 1 & 14.5 & 2.72 & 15 & 2.74 & 15.5 & 0.99 & 16.5 & 2.75 & 14 & 2467.21 & 22.5 & 3037.68 \\
\hline 28 & Heydarlou 1 & 10 & 3.00 & 11 & 3.00 & 11 & 1.20 & 12 & 3.01 & 9 & 2908.40 & 20 & 3103.55 \\
\hline 29 & Saribaglou 5 & 11 & 2.97 & 12 & 2.98 & 12 & 1.17 & 13 & 2.98 & 11 & 2853.26 & 19 & 3105.27 \\
\hline 30 & $\begin{array}{c}\text { Chongharalou-Yekan } \\
4\end{array}$ & 32 & 2.29 & 33.5 & 2.29 & 33.5 & 0.70 & 37 & 2.29 & 17 & 2344.98 & 42 & 2243.27 \\
\hline 31 & Maranghalou 6 & 8 & 3.05 & 10 & 3.05 & 10 & 1.23 & 10 & 3.05 & 5 & 3146.80 & 27 & 2961.98 \\
\hline 32 & Abajalou 1 & 3 & 3.55 & 3 & 3.55 & 3 & 1.67 & 4 & 3.55 & 3 & 3596.70 & 8 & 3505.17 \\
\hline 33 & Hamadan 1 & 6 & 3.26 & 7 & 3.26 & 6.5 & 1.41 & 6.5 & 3.27 & 6 & 3033.53 & 6 & 3513.70 \\
\hline 34 & Saghez 2 & 39.5 & 2.10 & 39.5 & 2.13 & 39.5 & 0.60 & 39 & 2.16 & 39 & 1799.07 & 37 & 2523.31 \\
\hline 35 & Piranshahr-Serokani & 49.5 & 1.48 & 50 & 1.48 & 50 & 0.29 & 51 & 1.48 & 45 & 1439.76 & 51 & 1529.26 \\
\hline 36 & Piranshahr Andizeh & 55 & 0.70 & 55 & 0.78 & 55 & 0.08 & 55 & 0.86 & 56 & 493.39 & 54 & 1222.34 \\
\hline 37 & Mashhad & 56 & 0.63 & 56 & 0.65 & 56 & 0.06 & 56 & 0.66 & 55 & 515.86 & 56 & 807.34 \\
\hline 38 & Shahroud 1 & 49.5 & 1.48 & 49 & 1.50 & 49 & 0.30 & 50 & 1.51 & 49 & 1315.93 & 49 & 1700.66 \\
\hline 39 & Hamadan 2 & 47 & 1.59 & 47 & 1.63 & 46.5 & 0.35 & 46 & 1.68 & 51 & 1285.08 & 44 & 2071.94 \\
\hline
\end{tabular}


192

\begin{tabular}{|c|c|c|c|c|c|c|c|c|c|c|c|c|c|}
\hline 40 & $\begin{array}{c}\text { Shabestar-Kouzeh- } \\
\text { Kanan } 3\end{array}$ & 51 & 1.37 & 52 & 1.37 & 52 & 0.25 & 52 & 1.38 & 48 & 1324.07 & 53 & 1426.11 \\
\hline 41 & Saghez 4 & 53 & 1.30 & 53 & 1.31 & 53 & 0.23 & 53 & 1.32 & 53 & 1177.20 & 52 & 1458.54 \\
\hline 42 & Saghez 5 & 37 & 2.13 & 37 & 2.24 & 37 & 0.66 & 33 & 2.34 & 41 & 1645.39 & 24 & 3036.84 \\
\hline 43 & Saghez 3 & 33.5 & 2.28 & 35.5 & 2.28 & 35.5 & 0.69 & 38 & 2.28 & 21 & 2183.06 & 39 & 2381.37 \\
\hline 44 & Shahroud 2 & 44 & 1.69 & 44 & 1.71 & 44 & 0.39 & 44.5 & 1.72 & 44 & 1502.04 & 46 & 1942.53 \\
\hline 45 & Alibaglou 1 & 38 & 2.12 & 39.5 & 2.13 & 39.5 & 0.60 & 40 & 2.14 & 31 & 1948.10 & 41 & 2331.95 \\
\hline 46 & Baneh 2 & 24 & 2.48 & 22 & 2.55 & 22 & 0.86 & 22 & 2.63 & 27 & 2009.26 & 16 & 3241.56 \\
\hline 47 & $\begin{array}{c}\text { Salmas-Gharagheshlagh- } \\
\text { Ghalami }\end{array}$ & 16 & 2.67 & 14 & 2.85 & 13.5 & 1.07 & 11 & 3.03 & 29 & 1987.24 & 2 & 4076.50 \\
\hline 48 & Marand-1389-2 & 33.5 & 2.28 & 28 & 2.40 & 28 & 0.77 & 24 & 2.54 & 40 & 1721.08 & 11 & 3356.18 \\
\hline 49 & $\begin{array}{c}\text { Salmas-Gharaghashlagh- } \\
\text { Badami }\end{array}$ & 7 & 3.13 & 8 & 3.14 & 8 & 1.30 & 8 & 3.14 & 8 & 2949.06 & 13 & 3340.35 \\
\hline 50 & $\begin{array}{c}\text { Shabestar-Kouzeh } \\
\text { Kanan } 1\end{array}$ & 29 & 2.35 & 32 & 2.35 & 32 & 0.73 & 32 & 2.35 & 18 & 2305.50 & 38 & 2390.03 \\
\hline 51 & Sanandaj & 29 & 2.35 & 29.5 & 2.38 & 29.5 & 0.75 & 30 & 2.40 & 25 & 2045.97 & 32 & 2756.99 \\
\hline 52 & $\begin{array}{c}\text { Shabestar-Kouzeh- } \\
\text { Kanan } 2\end{array}$ & 45 & 1.66 & 45 & 1.69 & 45 & 0.38 & 44.5 & 1.72 & 46 & 1403.79 & 45 & 2030.41 \\
\hline 53 & Baneh 3 & 36 & 2.26 & 35.5 & 2.28 & 35.5 & 0.69 & 36 & 2.30 & 30 & 1979.44 & 35 & 2624.47 \\
\hline 54 & Piranshahr-Baleban & 46 & 1.63 & 46 & 1.64 & 46.5 & 0.35 & 47 & 1.64 & 43 & 1522.93 & 48 & 1759.33 \\
\hline 55 & Baneh 1 & 26 & 2.37 & 26 & 2.44 & 26 & 0.79 & 27 & 2.52 & 34 & 1902.77 & 18 & 3141.23 \\
\hline 56 & Marand-1389-1 & 4.5 & 3.27 & 6 & 3.27 & 6.5 & 1.41 & 6.5 & 3.27 & 4 & 3228.56 & 14 & 3307.31 \\
\hline
\end{tabular}

Tab. 6. (Continued)

\begin{tabular}{|c|c|c|c|c|c|c|c|c|c|c|c|}
\hline No & Genotype & Rank & $\mathrm{M}_{\mathrm{S}} \mathrm{STI}$ & Rank & $\mathrm{M}_{\mathrm{P} S \mathrm{TI}}$ & Rank & $\mathrm{DI}$ & Rank & $\mathrm{YI}$ & Rank & SNPI \\
\hline 1 & Saghez 1 & 19 & 1.03 & 16 & 1.30 & 28 & 0.70 & 22 & 0.79 & 9 & 4.45 \\
\hline 2 & Anghane 4 & 16 & 1.16 & 1 & 7.97 & 53 & 0.25 & 38 & 0.66 & 1 & 9.08 \\
\hline 3 & Urmia-Barouj & 30 & 0.70 & 17 & 1.25 & 42 & 0.51 & 34.5 & 0.69 & 8 & 4.50 \\
\hline 4 & Urmia-Maranghalou & 41 & 0.39 & 31 & 0.77 & 50 & 0.42 & 42 & 0.59 & 21 & 3.68 \\
\hline 5 & Marand-Dizaj- Ghalami & 32 & 0.67 & 26 & 0.96 & 37.5 & 0.58 & 32.5 & 0.70 & 15 & 3.99 \\
\hline 6 & Jabalkandi 2 & 28 & 0.76 & 33 & 0.72 & 23 & 0.78 & 23 & 0.76 & 26 & 3.24 \\
\hline 7 & Salmas - Sadaghian & 18 & 1.07 & 18 & 1.24 & 25 & 0.75 & 19.5 & 0.81 & 12 & 4.32 \\
\hline 8 & Babaghanje 6 & 22 & 0.94 & 11 & 1.55 & 37.5 & 0.58 & 24.5 & 0.75 & 5 & 4.91 \\
\hline 9 & Miyaneh-Basin & 52 & 0.08 & 54 & 0.04 & 31.5 & 0.64 & 52 & 0.46 & 51 & -0.63 \\
\hline 10 & Boucan & 54 & 0.06 & 46 & 0.16 & 54 & 0.22 & 54 & 0.36 & 39 & 1.91 \\
\hline 11 & Urmia - Nuoshinshahr & 13 & 1.43 & 21 & 1.19 & 16 & 0.99 & 13 & 0.91 & 18 & 3.77 \\
\hline 12 & Karimabad & 2 & 5.46 & 2 & 3.28 & 3 & 1.70 & 2 & 1.32 & 23 & 3.46 \\
\hline 13 & Vaghaslou-Olya 1 & 43.5 & 0.20 & 39 & 0.49 & 52 & 0.31 & 48 & 0.48 & 29 & 3.06 \\
\hline 14 & Vaghaslou-Olya 3 & 11 & 2.11 & 9 & 2.02 & 15 & 1.00 & 12 & 0.98 & 4 & 5.02 \\
\hline 15 & Ordoshahi 1 & 21 & 0.96 & 27 & 0.90 & 21 & 0.83 & 19.5 & 0.81 & 22 & 3.56 \\
\hline 16 & Marana-Yamchi-Pesteii & 12 & 2.10 & 24 & 1.12 & 6 & 1.45 & 10 & 1.05 & 53 & -2.29 \\
\hline 17 & Mazandaran-Tirtash & 42 & 0.32 & 47.5 & 0.15 & 18 & 0.97 & 36 & 0.67 & 44.5 & 1.47 \\
\hline 18 & Sardasht & 39 & 0.44 & 42 & 0.42 & 29 & 0.68 & 38 & 0.66 & 34 & 2.59 \\
\hline 19 & Marana-Yamchi 4 & 49.5 & 0.12 & 49 & 0.13 & 46.5 & 0.46 & 50.5 & 0.47 & 42 & 1.65 \\
\hline 20 & Salmas 2 & 15 & 1.25 & 22 & 1.16 & 20 & 0.90 & 16.5 & 0.86 & 16 & 3.95 \\
\hline 21 & Vaghaslou-Olya 4 & 34 & 0.65 & 28 & 0.86 & 34 & 0.61 & 32.5 & 0.70 & 19.5 & 3.76 \\
\hline 22 & $\begin{array}{c}\text { Salmas-Gharaghashlagh- } \\
\text { Pesteii }\end{array}$ & 17 & 1.12 & 29.5 & 0.81 & 14 & 1.01 & 15 & 0.87 & 32 & 2.90 \\
\hline 23 & Lalalou-Torab 2 & 25 & 0.85 & 15 & 1.40 & 39 & 0.57 & 28 & 0.73 & 6 & 4.71 \\
\hline 24 & Shirabad 2 & 32 & 0.67 & 35 & 0.62 & 24 & 0.77 & 26 & 0.74 & 30 & 3.03 \\
\hline 25 & Gharagoz 1 & 1 & 5.92 & 3 & 2.91 & 1 & 1.96 & 1 & 1.38 & 56 & -4.57 \\
\hline 26 & Vaghaslou-Sofla 1 & 6 & 2.95 & 5 & 2.50 & 12 & 1.17 & 7.5 & 1.08 & 3 & 5.20 \\
\hline 27 & Khanneshan 1 & 14 & 1.42 & 19 & 1.21 & 18 & 0.97 & 14 & 0.90 & 17 & 3.87 \\
\hline 28 & Heydarlou 1 & 9 & 2.37 & 12 & 1.53 & 7 & 1.32 & 9 & 1.06 & 27 & 3.13 \\
\hline 29 & Saribaglou 5 & 10 & 2.24 & 13 & 1.50 & 8.5 & 1.27 & 11 & 1.04 & 25 & 3.36 \\
\hline 30 & Chongharalou-Yekan 4 & 24 & 0.90 & 40 & 0.46 & 11 & 1.19 & 16.5 & 0.86 & 52 & -1.78 \\
\hline 31 & Maranghalou 6 & 7 & 2.87 & 14 & 1.43 & 4 & 1.62 & 5 & 1.15 & 55 & -3.18 \\
\hline 32 & Abajalou 1 & 3 & 5.07 & 4 & 2.72 & 2 & 1.79 & 3 & 1.31 & 54 & -3.07 \\
\hline 33 & Hamadan 1 & 5 & 3.05 & 7 & 2.31 & 8.5 & 1.27 & 6 & 1.11 & 7 & 4.66 \\
\hline
\end{tabular}




\begin{tabular}{|c|c|c|c|c|c|c|c|c|c|c|c|}
\hline 34 & Saghez 2 & 38 & 0.46 & 38 & 0.51 & 33 & 0.62 & 38 & 0.66 & 31 & 2.94 \\
\hline 35 & Piranshahr-Serokani & 47.5 & 0.14 & 51 & 0.09 & 30 & 0.66 & 45 & 0.53 & 47 & 0.94 \\
\hline 36 & Piranshahr Andizeh & 55.5 & 0.00 & 55 & 0.02 & 56 & 0.10 & 56 & 0.18 & 49 & 0.72 \\
\hline 37 & Mashhad & 55.5 & 0.00 & 56 & 0.00 & 55 & 0.16 & 55 & 0.19 & 50 & 0.44 \\
\hline 38 & Shahroud 1 & 49.5 & 0.12 & 50 & 0.11 & 43 & 0.49 & 48 & 0.48 & 43 & 1.51 \\
\hline 39 & Hamadan 2 & 47.5 & 0.14 & 44 & 0.20 & 51 & 0.39 & 50.5 & 0.47 & 37 & 2.08 \\
\hline 40 & Shabestar-Kouzeh-Kanan 3 & 51 & 0.10 & 52 & 0.07 & 35.5 & 0.60 & 48 & 0.48 & 48 & 0.89 \\
\hline 41 & Saghez 4 & 53 & 0.07 & 53 & 0.06 & 46.5 & 0.46 & 53 & 0.43 & 46 & 1.14 \\
\hline 42 & Saghez 5 & 40 & 0.42 & 29.5 & 0.81 & 48.5 & 0.43 & 41 & 0.60 & 19.5 & 3.76 \\
\hline 43 & Saghez 3 & 27 & 0.77 & 37 & 0.52 & 18 & 0.97 & 21 & 0.80 & 36 & 2.17 \\
\hline 44 & Shahroud 2 & 43.5 & 0.20 & 45 & 0.19 & 40.5 & 0.56 & 44 & 0.55 & 40 & 1.88 \\
\hline 45 & Alibaglou 1 & 36 & 0.54 & 41 & 0.43 & 22 & 0.79 & 31 & 0.71 & 35 & 2.44 \\
\hline 46 & Baneh 2 & 26 & 0.82 & 20 & 1.20 & 35.5 & 0.60 & 28 & 0.73 & 10 & 4.38 \\
\hline 47 & $\begin{array}{c}\text { Salmas-Gharagheshlagh- } \\
\text { Ghalami }\end{array}$ & 20 & 0.99 & 6 & 2.36 & 44.5 & 0.47 & 28 & 0.73 & 2 & 5.88 \\
\hline 48 & Marand-1389-2 & 37 & 0.53 & 23 & 1.14 & 48.5 & 0.43 & 40 & 0.63 & 11 & 4.35 \\
\hline 49 & $\begin{array}{c}\text { Salmas-Gharaghashlagh- } \\
\text { Badami }\end{array}$ & 8 & 2.66 & 10 & 1.93 & 10 & 1.26 & 7.5 & 1.08 & 13.5 & 4.14 \\
\hline 50 & Shabestar-Kouzeh Kanan 1 & 23 & 0.91 & 36 & 0.55 & 13 & 1.08 & 18 & 0.84 & 41 & 1.71 \\
\hline 51 & Sanandaj & 29 & 0.73 & 32 & 0.75 & 26 & 0.74 & 24.5 & 0.75 & 24 & 3.40 \\
\hline 52 & Shabestar-Kouzeh-Kanan 2 & 46 & 0.17 & 43 & 0.21 & 44.5 & 0.47 & 46 & 0.51 & 38 & 2.05 \\
\hline 53 & Baneh 3 & 35 & 0.63 & 34 & 0.63 & 27 & 0.72 & 30 & 0.72 & 28 & 3.12 \\
\hline 54 & Piranshahr-Baleban & 45 & 0.19 & 47.5 & 0.15 & 31.5 & 0.64 & 43 & 0.56 & 44.5 & 1.47 \\
\hline 55 & Baneh 1 & 32 & 0.67 & 25 & 1.03 & 40.5 & 0.56 & 34.5 & 0.69 & 13.5 & 4.14 \\
\hline 56 & Marand-1389-1 & 4 & 3.46 & 8 & 2.05 & 5 & 1.53 & 4 & 1.18 & 33 & 2.61 \\
\hline
\end{tabular}

Tab. 7. Resistance/tolerance indices for 56 Iranian confectionery sunflower landraces under severe drought stressed and non-stressed conditions.

\begin{tabular}{|c|c|c|c|c|c|c|c|c|c|c|c|c|c|}
\hline No & Genotype & Rank & HARM & Rank & GMP & Rank & STI & Rank & MP & Rank & Ys-mild & Rank & $Y p$ \\
\hline 1 & Saghez 1 & 19 & 2.60 & 19.5 & 2.65 & 19.5 & 0.93 & 18.5 & 2.71 & 22 & 2169.72 & 15 & 3241.94 \\
\hline 2 & Anghane 4 & 13 & 2.81 & 4 & 3.38 & 4 & 1.51 & 1 & 4.06 & 38 & 1807.79 & 1 & 6310.77 \\
\hline 3 & Urmia-Barouj & 25 & 2.41 & 24.5 & 2.51 & 24 & 0.84 & 23 & 2.62 & 35 & 1883.44 & 12 & 3355.65 \\
\hline 4 & $\begin{array}{c}\text { Urmia- } \\
\text { Maranghalou }\end{array}$ & 39.5 & 2.10 & 38 & 2.20 & 38 & 0.64 & 34.5 & 2.31 & 42 & 1615.43 & 26 & 3001.82 \\
\hline 5 & $\begin{array}{c}\text { Marand-Dizaj- } \\
\text { Ghalami }\end{array}$ & 27 & 2.36 & 27 & 2.42 & 27 & 0.78 & 28 & 2.49 & 33 & 1917.77 & 21 & 3055.23 \\
\hline 6 & Jabalkandi 2 & 29 & 2.35 & 31 & 2.37 & 31 & 0.74 & 31 & 2.39 & 23 & 2085.56 & 33 & 2695.38 \\
\hline 7 & Salmas - Sadaghian & 18 & 2.61 & 19.5 & 2.65 & 19.5 & 0.93 & 21 & 2.69 & 19 & 2211.75 & 17 & 3177.21 \\
\hline 8 & Babaghanje 6 & 20 & 2.59 & 17.5 & 2.68 & 17.5 & 0.95 & 15 & 2.78 & 24 & 2048.18 & 7 & 3505.74 \\
\hline 9 & Miyaneh-Basin & 54 & 1.25 & 54 & 1.25 & 54 & 0.21 & 54 & 1.25 & 52 & 1273.47 & 55 & 1221.97 \\
\hline 10 & Boucan & 52 & 1.33 & 51 & 1.43 & 51 & 0.27 & 48.5 & 1.54 & 54 & 973.88 & 43 & 2103.75 \\
\hline 11 & $\begin{array}{c}\text { Urmia - } \\
\text { Nuoshinshahr }\end{array}$ & 14.5 & 2.72 & 16 & 2.73 & 15.5 & 0.99 & 16.5 & 2.75 & 13 & 2482.86 & 25 & 3012.03 \\
\hline 12 & Karimabad & 1 & 3.67 & 1 & 3.67 & 1 & 1.78 & 2 & 3.67 & 2 & 3611.45 & 3 & 3728.20 \\
\hline 13 & Vaghaslou-Olya 1 & 42 & 1.80 & 42 & 1.92 & 42 & 0.49 & 42 & 2.05 & 47 & 1328.74 & 31 & 2768.42 \\
\hline 14 & Vaghaslou-Olya 3 & 9 & 3.04 & 9 & 3.07 & 9 & 1.25 & 9 & 3.09 & 12 & 2686.43 & 9 & 3500.27 \\
\hline 15 & Ordoshahi 1 & 23 & 2.49 & 24.5 & 2.51 & 25 & 0.83 & 25.5 & 2.53 & 20 & 2208.24 & 29 & 2852.48 \\
\hline 16 & $\begin{array}{c}\text { Marana-Yamchi- } \\
\text { Pesteii }\end{array}$ & 12 & 2.85 & 13 & 2.85 & 13.5 & 1.07 & 14 & 2.85 & 10 & 2889.97 & 30 & 2802.96 \\
\hline 17 & $\begin{array}{c}\text { Mazandaran- } \\
\text { Tirtash }\end{array}$ & 43 & 1.75 & 43 & 1.75 & 43 & 0.41 & 43 & 1.75 & 36 & 1832.44 & 50 & 1675.26 \\
\hline 18 & Sardasht & 41 & 2.05 & 41 & 2.07 & 41 & 0.57 & 41 & 2.09 & 37 & 1816.67 & 40 & 2356.11 \\
\hline 19 & Marana-Yamchi 4 & 48 & 1.50 & 48 & 1.52 & 48 & 0.31 & 48.5 & 1.54 & 50 & 1299.26 & 47 & 1783.27 \\
\hline 20 & Salmas 2 & 17 & 2.66 & 17.5 & 2.68 & 17.5 & 0.95 & 20 & 2.70 & 16 & 2367.56 & 22.5 & 3037.67 \\
\hline 21 & Vaghaslou-Olya 4 & 31 & 2.33 & 29.5 & 2.38 & 29.5 & 0.75 & 29 & 2.43 & 32 & 1927.74 & 28 & 2935.50 \\
\hline 22 & $\begin{array}{l}\text { Salmas- } \\
\text { Gharaghashlagh- } \\
\text { Pesteii }\end{array}$ & 22 & 2.52 & 23 & 2.53 & 23 & 0.85 & 25.5 & 2.53 & 15 & 2371.90 & 34 & 2691.73 \\
\hline 23 & Lalalou-Torab 2 & 21 & 2.53 & 21 & 2.62 & 21 & 0.91 & 18.5 & 2.71 & 28 & 2002.13 & 10 & 3418.40 \\
\hline 24 & Shirabad 2 & 35 & 2.27 & 33.5 & 2.29 & 33.5 & 0.70 & 34.5 & 2.31 & 26 & 2026.19 & 36 & 2591.75 \\
\hline
\end{tabular}


194

\begin{tabular}{|c|c|c|c|c|c|c|c|c|c|c|c|c|c|}
\hline 25 & Gharagoz 1 & 2 & 3.65 & 2 & 3.65 & 2 & 1.77 & 3 & 3.65 & 1 & 3778.83 & 5 & 3528.58 \\
\hline 26 & Vaghaslou-Sofla 1 & 4.5 & 3.27 & 5 & 3.28 & 5 & 1.43 & 5 & 3.30 & 7 & 2965.42 & 4 & 3635.39 \\
\hline 27 & Khanneshan 1 & 14.5 & 2.72 & 15 & 2.74 & 15.5 & 0.99 & 16.5 & 2.75 & 14 & 2467.21 & 22.5 & 3037.68 \\
\hline 28 & Heydarlou 1 & 10 & 3.00 & 11 & 3.00 & 11 & 1.20 & 12 & 3.01 & 9 & 2908.40 & 20 & 3103.55 \\
\hline 29 & Saribaglou 5 & 11 & 2.97 & 12 & 2.98 & 12 & 1.17 & 13 & 2.98 & 11 & 2853.26 & 19 & 3105.27 \\
\hline 30 & $\begin{array}{l}\text { Chongharalou- } \\
\text { Yekan } 4\end{array}$ & 32 & 2.29 & 33.5 & 2.29 & 33.5 & 0.70 & 37 & 2.29 & 17 & 2344.98 & 42 & 2243.27 \\
\hline 31 & Maranghalou 6 & 8 & 3.05 & 10 & 3.05 & 10 & 1.23 & 10 & 3.05 & 5 & 3146.80 & 27 & 2961.98 \\
\hline 32 & Abajalou 1 & 3 & 3.55 & 3 & 3.55 & 3 & 1.67 & 4 & 3.55 & 3 & 3596.70 & 8 & 3505.17 \\
\hline 33 & Hamadan 1 & 6 & 3.26 & 7 & 3.26 & 6.5 & 1.41 & 6.5 & 3.27 & 6 & 3033.53 & 6 & 3513.70 \\
\hline 34 & Saghez 2 & 39.5 & 2.10 & 39.5 & 2.13 & 39.5 & 0.60 & 39 & 2.16 & 39 & 1799.07 & 37 & 2523.31 \\
\hline 35 & $\begin{array}{l}\text { Piranshahr- } \\
\text { Serokani }\end{array}$ & 49.5 & 1.48 & 50 & 1.48 & 50 & 0.29 & 51 & 1.48 & 45 & 1439.76 & 51 & 1529.26 \\
\hline 36 & $\begin{array}{l}\text { Piranshahr } \\
\text { Andizeh }\end{array}$ & 55 & 0.70 & 55 & 0.78 & 55 & 0.08 & 55 & 0.86 & 56 & 493.39 & 54 & 1222.34 \\
\hline 37 & Mashhad & 56 & 0.63 & 56 & 0.65 & 56 & 0.06 & 56 & 0.66 & 55 & 515.86 & 56 & 807.34 \\
\hline 38 & Shahroud 1 & 49.5 & 1.48 & 49 & 1.50 & 49 & 0.30 & 50 & 1.51 & 49 & 1315.93 & 49 & 1700.66 \\
\hline 39 & Hamadan 2 & 47 & 1.59 & 47 & 1.63 & 46.5 & 0.35 & 46 & 1.68 & 51 & 1285.08 & 44 & 2071.94 \\
\hline 40 & $\begin{array}{c}\text { Shabestar-Kouzeh- } \\
\text { Kanan } 3\end{array}$ & 51 & 1.37 & 52 & 1.37 & 52 & 0.25 & 52 & 1.38 & 48 & 1324.07 & 53 & 1426.11 \\
\hline 41 & Saghez 4 & 53 & 1.30 & 53 & 1.31 & 53 & 0.23 & 53 & 1.32 & 53 & 1177.20 & 52 & 1458.54 \\
\hline 42 & Saghez 5 & 37 & 2.13 & 37 & 2.24 & 37 & 0.66 & 33 & 2.34 & 41 & 1645.39 & 24 & 3036.84 \\
\hline 43 & Saghez 3 & 33.5 & 2.28 & 35.5 & 2.28 & 35.5 & 0.69 & 38 & 2.28 & 21 & 2183.06 & 39 & 2381.37 \\
\hline 44 & Shahroud 2 & 44 & 1.69 & 44 & 1.71 & 44 & 0.39 & 44.5 & 1.72 & 44 & 1502.04 & 46 & 1942.53 \\
\hline 45 & Alibaglou 1 & 38 & 2.12 & 39.5 & 2.13 & 39.5 & 0.60 & 40 & 2.14 & 31 & 1948.10 & 41 & 2331.95 \\
\hline 46 & Baneh 2 & 24 & 2.48 & 22 & 2.55 & 22 & 0.86 & 22 & 2.63 & 27 & 2009.26 & 16 & 3241.56 \\
\hline 47 & $\begin{array}{l}\text { Salmas- } \\
\text { Gharagheshlagh- } \\
\text { Ghalami }\end{array}$ & 16 & 2.67 & 14 & 2.85 & 13.5 & 1.07 & 11 & 3.03 & 29 & 1987.24 & 2 & 4076.50 \\
\hline 48 & Marand-1389-2 & 33.5 & 2.28 & 28 & 2.40 & 28 & 0.77 & 24 & 2.54 & 40 & 1721.08 & 11 & 3356.18 \\
\hline 49 & $\begin{array}{c}\text { Salmas- } \\
\text { Gharaghashlagh- } \\
\text { Badami }\end{array}$ & 7 & 3.13 & 8 & 3.14 & 8 & 1.30 & 8 & 3.14 & 8 & 2949.06 & 13 & 3340.35 \\
\hline 50 & $\begin{array}{c}\text { Shabestar-Kouzeh } \\
\text { Kanan } 1\end{array}$ & 29 & 2.35 & 32 & 2.35 & 32 & 0.73 & 32 & 2.35 & 18 & 2305.50 & 38 & 2390.03 \\
\hline 51 & Sanandaj & 29 & 2.35 & 29.5 & 2.38 & 29.5 & 0.75 & 30 & 2.40 & 25 & 2045.97 & 32 & 2756.99 \\
\hline 52 & $\begin{array}{c}\text { Shabestar-Kouzeh- } \\
\text { Kanan } 2\end{array}$ & 45 & 1.66 & 45 & 1.69 & 45 & 0.38 & 44.5 & 1.72 & 46 & 1403.79 & 45 & 2030.41 \\
\hline 53 & Baneh 3 & 36 & 2.26 & 35.5 & 2.28 & 35.5 & 0.69 & 36 & 2.30 & 30 & 1979.44 & 35 & 2624.47 \\
\hline 54 & Piranshahr-Baleban & 46 & 1.63 & 46 & 1.64 & 46.5 & 0.35 & 47 & 1.64 & 43 & 1522.93 & 48 & 1759.33 \\
\hline 55 & Baneh 1 & 26 & 2.37 & 26 & 2.44 & 26 & 0.79 & 27 & 2.52 & 34 & 1902.77 & 18 & 3141.23 \\
\hline 56 & Marand-1389-1 & 4.5 & 3.27 & 6 & 3.27 & 6.5 & 1.41 & 6.5 & 3.27 & 4 & 3228.56 & 14 & 3307.31 \\
\hline
\end{tabular}

Tab. 7. (Continued)

\begin{tabular}{|c|c|c|c|c|c|c|c|c|c|c|c|}
\hline No & Genotype & Rank & $\mathrm{M}_{\mathrm{S}} \mathrm{STI}$ & Rank & $\mathrm{M}_{\mathrm{P}} \mathrm{STI}$ & Rank & DI & Rank & YI & Rank & SNPI \\
\hline 1 & Saghez 1 & 31 & 0.46 & 22.5 & 0.76 & 40.5 & 0.36 & 35 & 0.46 & 36.5 & 2.29 \\
\hline 2 & Anghane 4 & 8 & 1.34 & 1 & 6.44 & 46.5 & 0.24 & 25 & 0.53 & 22 & 2.78 \\
\hline 3 & Urmia-Barouj & 18 & 0.90 & 11 & 1.04 & 28.5 & 0.53 & 19.5 & 0.57 & 21 & 2.84 \\
\hline 4 & Urmia-Maranghalou & 36.5 & 0.33 & 28 & 0.56 & 43 & 0.33 & 39.5 & 0.43 & 39 & 2.11 \\
\hline 5 & Marand-Dizaj- Ghalami & 3 & 1.84 & 12.5 & 1.03 & 4 & 1.00 & 3 & 0.75 & 3 & 4.06 \\
\hline 6 & Jabalkandi 2 & 30 & 0.52 & 35 & 0.49 & 28.5 & 0.53 & 30.5 & 0.51 & 30 & 2.59 \\
\hline 7 & Salmas - Sadaghian & 1 & 2.37 & 5 & 1.25 & 3 & 1.11 & 1 & 0.81 & 2 & 4.44 \\
\hline 8 & Babaghanje 6 & 43 & 0.24 & 22.5 & 0.76 & 52.5 & 0.21 & 47 & 0.36 & 46 & 1.85 \\
\hline 9 & Miyaneh-Basin & 40.5 & 0.26 & 51.5 & 0.05 & 2 & 1.26 & 25 & 0.53 & 56 & -3.08 \\
\hline 10 & Boucan & 51 & 0.07 & 46 & 0.13 & 54 & 0.20 & 52 & 0.28 & 51 & 1.39 \\
\hline 11 & Urmia - Nuoshinshahr & 13 & 1.17 & 19 & 0.85 & 12.5 & 0.76 & 11.5 & 0.65 & 11 & 3.34 \\
\hline 12 & Karimabad & 2 & 1.98 & 2 & 1.80 & 12.5 & 0.76 & 5.5 & 0.72 & 8 & 3.63 \\
\hline 13 & Vaghaslou-Olya 1 & 27.5 & 0.56 & 30 & 0.54 & 26 & 0.54 & 28.5 & 0.52 & 25 & 2.63 \\
\hline 14 & Vaghaslou-Olya 3 & 23 & 0.73 & 9 & 1.09 & 35.5 & 0.43 & 25 & 0.53 & 28 & 2.61 \\
\hline 15 & Ordoshahi 1 & 19 & 0.82 & 26 & 0.66 & 19.5 & 0.66 & 17 & 0.59 & 19 & 2.98 \\
\hline
\end{tabular}




\begin{tabular}{|c|c|c|c|c|c|c|c|c|c|c|c|}
\hline 16 & Marana-Yamchi-Pesteii & 27.5 & 0.56 & 29 & 0.55 & 28.5 & 0.53 & 28.5 & 0.52 & 28 & 2.61 \\
\hline 17 & Mazandaran-Tirtash & 49 & 0.11 & 49.5 & 0.08 & 35.5 & 0.43 & 47 & 0.36 & 45 & 1.87 \\
\hline 18 & Sardasht & 29 & 0.54 & 39 & 0.34 & 17 & 0.69 & 21 & 0.55 & 20 & 2.88 \\
\hline 19 & Marana-Yamchi 4 & 45.5 & 0.20 & 47 & 0.12 & 25 & 0.56 & 39.5 & 0.43 & 36.5 & 2.29 \\
\hline 20 & Salmas 2 & 47 & 0.19 & 37 & 0.47 & 50.5 & 0.22 & 49 & 0.35 & 48 & 1.76 \\
\hline 21 & Vaghaslou-Olya 4 & 17 & 0.97 & 24 & 0.75 & 14.5 & 0.70 & 15.5 & 0.62 & 15.5 & 3.15 \\
\hline 22 & $\begin{array}{c}\text { Salmas-Gharaghashlagh- } \\
\text { Pesteii }\end{array}$ & 11 & 1.26 & 27 & 0.65 & 5 & 0.97 & 7 & 0.69 & 6 & 3.83 \\
\hline 23 & Lalalou-Torab 2 & 39 & 0.27 & 25 & 0.74 & 48.5 & 0.23 & 45 & 0.38 & 44 & 1.93 \\
\hline 24 & Shirabad 2 & 24 & 0.71 & 35 & 0.49 & 14.5 & 0.70 & 18 & 0.58 & 18 & 3.01 \\
\hline 25 & Gharagoz 1 & 10 & 1.28 & 3.5 & 1.35 & 22 & 0.62 & 13 & 0.64 & 13 & 3.17 \\
\hline 26 & Vaghaslou-Sofla 1 & 20 & 0.79 & 6 & 1.24 & 35.5 & 0.43 & 25 & 0.53 & 24 & 2.65 \\
\hline 27 & Khanneshan 1 & 15 & 1.03 & 20.5 & 0.84 & 17 & 0.69 & 15.5 & 0.62 & 15.5 & 3.15 \\
\hline 28 & Heydarlou 1 & 45.5 & 0.20 & 32 & 0.51 & 50.5 & 0.22 & 47 & 0.36 & 47 & 1.79 \\
\hline 29 & Saribaglou 5 & 4 & 1.78 & 10 & 1.07 & 6.5 & 0.96 & 4 & 0.74 & 4 & 3.94 \\
\hline 30 & Chongharalou-Yekan 4 & 26 & 0.59 & 41.5 & 0.31 & 9 & 0.79 & 19.5 & 0.57 & 17 & 3.14 \\
\hline 31 & Maranghalou 6 & 6 & 1.58 & 16.5 & 0.91 & 6.5 & 0.96 & 5.5 & 0.72 & 5 & 3.89 \\
\hline 32 & Abajalou 1 & 36.5 & 0.33 & 20.5 & 0.84 & 45 & 0.26 & 42.5 & 0.41 & 42 & 2.03 \\
\hline 33 & Hamadan 1 & 22 & 0.74 & 8 & 1.11 & 35.5 & 0.43 & 25 & 0.53 & 28 & 2.61 \\
\hline 34 & Saghez 2 & 16 & 1.02 & 32 & 0.51 & 8 & 0.93 & 9.5 & 0.66 & 7 & 3.68 \\
\hline 35 & Piranshahr-Serokani & 51 & 0.07 & 51.5 & 0.05 & 42 & 0.35 & 50 & 0.31 & 49 & 1.59 \\
\hline 36 & Piranshahr Andizeh & 55 & 0.01 & 55 & 0.02 & 55 & 0.15 & 55.5 & 0.18 & 55 & 0.90 \\
\hline 37 & Mashhad & 55 & 0.01 & 56 & 0.00 & 52.5 & 0.21 & 55.5 & 0.18 & 54 & 0.93 \\
\hline 38 & Shahroud 1 & 44 & 0.22 & 48 & 0.11 & 21 & 0.65 & 36 & 0.45 & 31 & 2.56 \\
\hline 39 & Hamadan 2 & 40.5 & 0.26 & 43 & 0.19 & 31 & 0.51 & 37.5 & 0.44 & 38 & 2.28 \\
\hline 40 & Shabestar-Kouzeh-Kanan 3 & 53 & 0.03 & 53 & 0.04 & 46.5 & 0.24 & 53 & 0.25 & 52 & 1.25 \\
\hline 41 & Saghez 4 & 55 & 0.01 & 54 & 0.03 & 56 & 0.14 & 54 & 0.19 & 53 & 0.95 \\
\hline 42 & Saghez 5 & 12 & 1.21 & 18 & 0.89 & 11 & 0.77 & 9.5 & 0.66 & 10 & 3.38 \\
\hline 43 & Saghez 3 & 5 & 1.60 & 32 & 0.51 & 1 & 1.39 & 2 & 0.78 & 1 & 5.91 \\
\hline 44 & Shahroud 2 & 48 & 0.16 & 45 & 0.14 & 38 & 0.42 & 44 & 0.39 & 43 & 1.95 \\
\hline 45 & Alibaglou 1 & 33 & 0.43 & 41.5 & 0.31 & 23 & 0.60 & 30.5 & 0.51 & 26 & 2.62 \\
\hline 46 & Baneh 2 & 14 & 1.14 & 12.5 & 1.03 & 19.5 & 0.66 & 14 & 0.63 & 14 & 3.16 \\
\hline 47 & $\begin{array}{c}\text { Salmas-Gharagheshlagh- } \\
\text { Ghalami }\end{array}$ & 35 & 0.41 & 3.5 & 1.35 & 48.5 & 0.23 & 42.5 & 0.41 & 40 & 2.10 \\
\hline 48 & Marand-1389-2 & 25 & 0.60 & 16.5 & 0.91 & 39 & 0.41 & 32.5 & 0.50 & 33 & 2.48 \\
\hline 49 & $\begin{array}{c}\text { Salmas-Gharaghashlagh- } \\
\text { Badami }\end{array}$ & 9 & 1.30 & 7 & 1.17 & 17 & 0.69 & 11.5 & 0.65 & 12 & 3.28 \\
\hline 50 & Shabestar-Kouzeh Kanan 1 & 34 & 0.42 & 40 & 0.33 & 24 & 0.57 & 32.5 & 0.50 & 32 & 2.55 \\
\hline 51 & Sanandaj & 32 & 0.45 & 35 & 0.49 & 33 & 0.47 & 34 & 0.49 & 34 & 2.42 \\
\hline 52 & Shabestar-Kouzeh-Kanan 2 & 42 & 0.25 & 44 & 0.18 & 28.5 & 0.53 & 37.5 & 0.44 & 35 & 2.30 \\
\hline 53 & Baneh 3 & 38 & 0.28 & 38 & 0.37 & 40.5 & 0.36 & 41 & 0.42 & 41 & 2.08 \\
\hline 54 & Piranshahr-Baleban & 51 & 0.07 & 49.5 & 0.08 & 44 & 0.28 & 51 & 0.30 & 50 & 1.50 \\
\hline 55 & Baneh 1 & 7 & 1.35 & 14 & 1.00 & 10 & 0.78 & 8 & 0.67 & 9 & 3.45 \\
\hline 56 & Marand-1389-1 & 21 & 0.75 & 15 & 0.95 & 32 & 0.48 & 22 & 0.54 & 23 & 2.68 \\
\hline
\end{tabular}

such as MP, STI, GMP, HARM, SNPI, YI, DI and $\mathrm{M}_{S} S T I$ were the best predictors of $Y_{P}$ and $Y_{S}$ than other indices. The observed relationship between YP, MP, STI, YS, MP and STI are in consistent with those reported by Fernansez (1992) in mungbean and Farshadfar et al. (2013) in wheat. Toorchi et al. (2012) showed that correlation between MP, GMP and YS and YP is positive. Ebrahimzadeh Banayjedi et al. (2012) introduced STI, MP and GMP as the best indices for yield predicting. Dehghani et al. (2009) reported that GMP, MP STI were significantly and positively correlated with stress yield. Farshadfar et al. (2001) showed that the most appropriate index for selecting stress tolerant cultivars is an index which has partly high correlation with grain yield under stress and non-stressed conditions. There were negative and significant correlations between YP and YSI in moderate and severe drought stressed conditions.

\section{Correlation analysis}

Based on cluster analysis, the studied landraces were grouped into 3 clusters in mild and severe drought stressed conditions. Therefore, there was considerable variation among landraces for drought tolerance. The dendrogram of accessions did not divide the accessions into distinct groups resembling the similar geographical distribution (Fig. 1 and 2). Therefore, it is suggested that selection of parents for hybridization does not need to be based on geographic diversity. Hybridization/crossing between any distantly related populations is expected to yield more heterosis and vigorous plants. According to the dendrogram 1,30\% of landraces situated in groups $1,34 \%$ situated in groups 2 and $36 \%$ in group 3 under mild drought stressed conditions 
196

(Fig. 1). In severe drought stressed conditions, $30 \%$ of landraces placed in groups $1,21 \%$ situated in groups 2 and $45 \%$ in group 3 (Fig. 2). Cluster analysis has been used in drought tolerance studies by other researchers (Safahani Langroodi et al., 2013; Dehbalaei et al., 2013; Zaheri and Bahraminejad, 2012; Ajalli and Salehi, 2012; Zare, 2012; Tabatabaei, 2013). Golabadi et al. (2006) in characterizing durum wheat segregating populations with cluster analysis distinguished groups with superior lines in both drought stress and well-watered conditions, superior lines in only drought stressed conditions as well as superior lines only in well-watered conditions considering their grain yield. Sajad Bakaei et al. (2008) showed that based on cluster analysis, in normal conditions genotypes were divided into two groups while in mild and severe stressed conditions genotypes were divided into 4 and 5 groups, respectively. Based on results of Zahravi (2009) regarded to cluster analysis, genotypes were divided into three groups resistant, semi-resistant and susceptible.

Using the biplot diagram (Fig. 3) the landraces 2, 12, 14, $25,26,28,31,32,33,49$ and 56 under mild stressed conditions were identified as tolerant and the landraces 9 , $10,35,36,37,38,40$ and 41 were detected as sensitive to drought. In severe stressed conditions the landraces 2, 5, 7 , 12,25 and 29 were identified as tolerant and the landraces
$10,35,36,37,40$ and 41 were detected as sensitive to drought (Fig 4). According to the biplot presentation there is positive correlation between indices MP, GMP, HARM, STI and yield in three environments that, confirm the observed simple correlations between them. Accordingly, in this study four above indices are the most appropriate indices to screening drought tolerant genotypes. The results of this study are compatible with Fernandez (1992), Golabadi et al. (2006), Kaya et al. (2002) and Farshadfar et al. (2012).

\section{Conclusion}

Abiotic stress tolerance is a key component and in some cases the major factor in improving yield in crops (Tollenaar and $\mathrm{Wu}, 1999$ ). Drought stress is considered as one of the most visible factors, which affect grain yield, and some of the constituents of the grain oil of sunflower (Razi and Assad, 1999; Ali et al., 2009). In this evaluation, based on cluster analysis, the studied genotypes were grouped in 3 clusters in each one of water treatment conditions. To select drought tolerant genotypes in severe drought stress conditions, it is suggested to use indices MP, STI, GMP, HARM, SNPI and MsSTI because these indices supports stable and high yield in both non-stress and stress conditions.



Population

Fig. 1. Cluster diagram Dendrogram of 56 Iranian sunflower landraces generated by Wards clustering method by square Euclidean distance after data normalization based on drought tolerance indices (MP, STI, GMP, HARM, SNPI, YI, DI and $\mathrm{M}_{S} \mathrm{STI}$ ) in moderate drought stress conditions 




Fig. 2. Cluster diagram Dendrogram of 56 Iranian sunflower landraces generated by Wards clustering method by square Euclidean distance after data normalization based on drought tolerance indices (MP, STI, GMP, HARM, SNPI, YI, DI and $\left.\mathrm{M}_{S} \mathrm{STI}\right)$ in severe drought stress conditions.

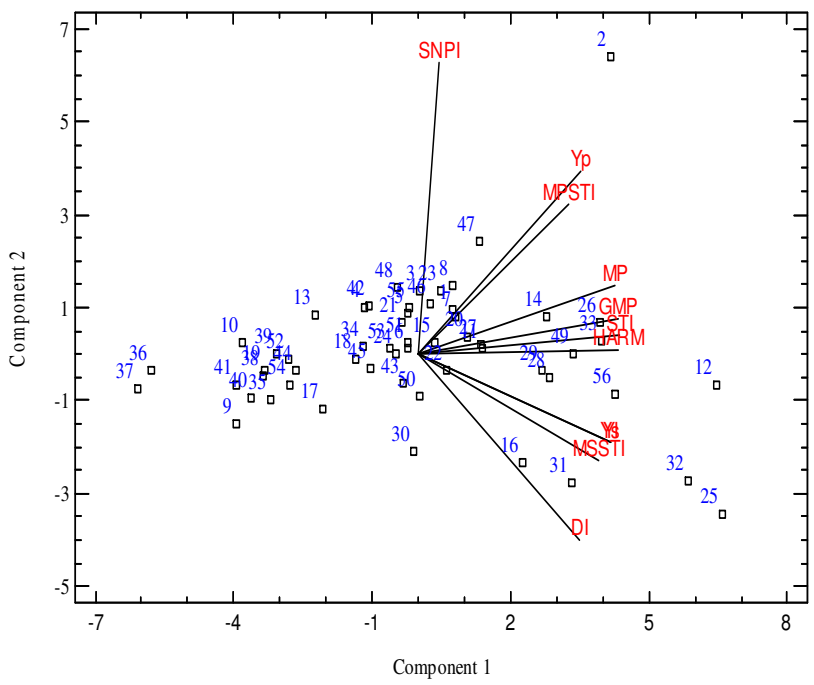

Fig. 3. Biplot for determination of the superior indices and genotypes in 56 Iranian sunflower landraces in moderate drought stress conditions.

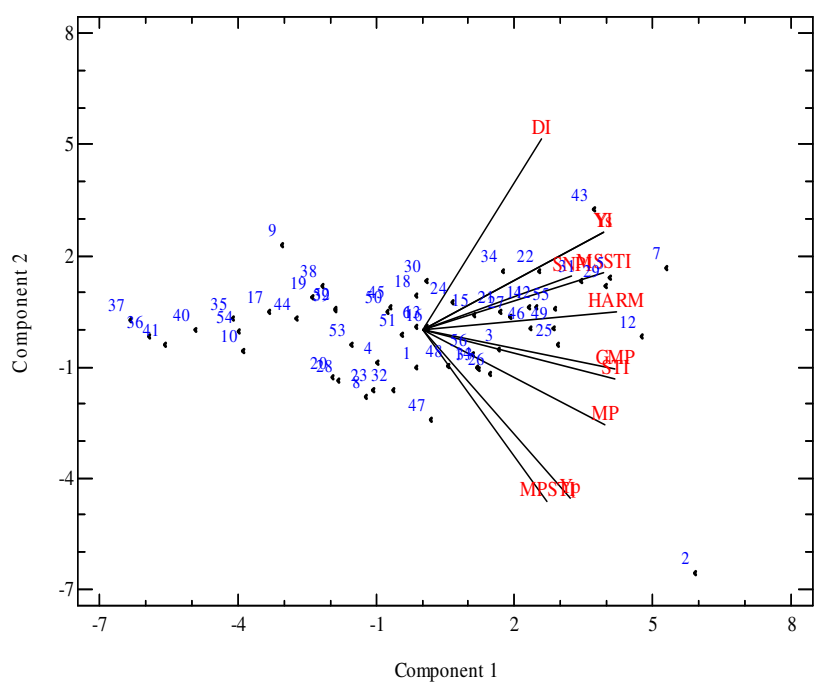

Fig. 4. Biplot for determination of the superior indices and genotypes in 56 Iranian sunflower landraces in severe drought stress conditions. 
198

Tab. 8. Correlation coefficients between drought tolerance indices and grain yield in both moderate and sever drought stress conditions

\begin{tabular}{|c|c|c|c|c|c|c|c|c|c|c|c|}
\hline & $\mathrm{YP}$ & YS & MP & STI & GMP & HARM & SNPI & $\mathrm{YI}$ & DI & MPSTI & MSSTI \\
\hline YP & 1 & & & & & & & & & & \\
\hline YS & $0.58^{* \prime}$ & 1 & & & & & & & & & \\
\hline MP & $0.91^{*}$ & $0.86^{*}$ & 1 & & & & & & & & \\
\hline STI & $0.82^{*}$ & $0.91^{*}$ & $0.97^{*}$ & 1 & & & & & & & \\
\hline GMP & $0.85^{*}$ & $0.91^{*}$ & $0.99^{* *}$ & $0.98^{* *}$ & 1 & & & & & & \\
\hline HARM & $0.79^{*}$ & $0.95^{*}$ & $0.96^{*}$ & $0.97^{*}$ & $0.99^{* *}$ & 1 & & & & & \\
\hline SNPI & $0.53^{*}$ & $-0.10^{\text {ns }}$ & $0.28^{*}$ & $0.14^{\mathrm{ns}}$ & $0.21^{\mathrm{ns}}$ & $0.15^{\text {ns }}$ & 1 & & & & \\
\hline YI & $0.58^{*}$ & $0.99^{*}$ & $0.86^{*}$ & $0.91^{*}$ & $0.91^{* *}$ & $0.95^{*}$ & $-0.09^{\mathrm{ns}}$ & 1 & & & \\
\hline DI & $0.28^{*}$ & $0.93^{*}$ & $0.64^{\circ}$ & $0.75^{*}$ & $0.72^{* *}$ & $0.78^{* \prime}$ & $-0.40^{* *}$ & $0.93^{* *}$ & 1 & & \\
\hline MPSTI & $0.88^{*}$ & $0.52^{*}$ & $0.80^{* \prime}$ & $0.78^{*}$ & $0.73^{* *}$ & $0.66^{\prime \prime}$ & $0.37^{*}$ & $0.52^{*}$ & $0.31^{*}$ & 1 & \\
\hline MSSTI & $0.51^{*}$ & $0.90^{* *}$ & $0.77^{* \prime}$ & $0.88^{* *}$ & $0.81^{* *}$ & $0.83^{\prime \prime}$ & $-0.23^{\mathrm{ns}}$ & $0.90^{* *}$ & $0.87^{*}$ & $0.57^{* *}$ & 1 \\
\hline
\end{tabular}

Tab. 9. Correlation coefficients between drought tolerance indices and grain yield in both optimum and sever drought stress conditions

\begin{tabular}{|c|c|c|c|c|c|c|c|c|c|c|c|}
\hline & YP & YS & MP & STI & GMP & HARM & SNPI & YI & DI & MPSTI & MSSTI \\
\hline YP & 1 & & & & & & & & & & \\
\hline YS & $0.45^{* *}$ & 1 & & & & & & & & & \\
\hline MP & $0.94^{* *}$ & $0.72^{*}$ & 1 & & & & & & & & \\
\hline STI & $0.84^{* *}$ & $0.83^{*}$ & $0.96^{\prime \prime}$ & 1 & & & & & & & \\
\hline GMP & $0.84^{* *}$ & $0.85^{*}$ & $0.97^{\prime \prime}$ & $0.98^{* \prime}$ & 1 & & & & & & \\
\hline HARM & $0.70^{* *}$ & $0.94^{*}$ & $0.89^{*}$ & $0.95^{\prime \prime}$ & $0.97^{\prime \prime}$ & 1 & & & & & \\
\hline SNPI & $0.42^{* *}$ & $0.72^{*}$ & $0.59^{* \prime}$ & $0.67^{* \prime}$ & $0.69^{* \prime}$ & $0.74^{* *}$ & 1 & & & & \\
\hline YI & $0.45^{* *}$ & $0.99^{*}$ & $0.71^{\prime \prime}$ & $0.83^{\prime \prime}$ & $0.85^{\prime \prime}$ & $0.94^{\prime \prime}$ & $0.72^{\prime \prime}$ & 1 & & & \\
\hline DI & $-0.02^{\mathrm{ns}}$ & $0.84^{*}$ & $0.29^{*}$ & $0.45^{\prime \prime}$ & $0.47^{\prime \prime}$ & $0.62^{* *}$ & $0.46^{*}$ & $0.84^{*}$ & 1 & & \\
\hline MPSTI & $0.84^{* *}$ & $0.33^{*}$ & $0.77^{*}$ & $0.74^{\prime \prime}$ & $0.66^{\prime \prime}$ & $0.52^{* *}$ & $0.27^{*}$ & $0.33^{*}$ & $-0.008^{\mathrm{ns}}$ & 1 & \\
\hline MSSTI & $0.52^{* *}$ & $0.91^{*}$ & $0.74^{*}$ & $0.88^{* \prime}$ & $0.84^{*}$ & $0.89^{* \prime}$ & $0.74^{* \prime}$ & $0.91^{*}$ & $0.70^{* *}$ & $0.48^{* \prime}$ & 1 \\
\hline
\end{tabular}

Tab 10. Ranking of 56 Iranian confectionery sunflower landraces based on the best indices in optimum and stress drought stressed conditions

\begin{tabular}{|c|c|c|c|c|c|c|}
\hline \multirow{2}{*}{ No } & \multirow{2}{*}{ Genotype } & \multicolumn{3}{|c|}{ Yield } & \multirow{2}{*}{$\begin{array}{c}\text { Rank } \\
\text { Normal-severe } \\
\text { stress }\end{array}$} & \multirow{2}{*}{$\begin{array}{l}\text { Normal-mild } \\
\text { stress }\end{array}$} \\
\hline & & Ys-severe & YS - mild & YP & & \\
\hline 1 & Saghez 1 & 1272.33 & 2169.72 & 3241.94 & 26.5 & 19.5 \\
\hline 2 & Anghane 4 & 1461.19 & 1807.79 & 6310.77 & 1 & 4 \\
\hline 3 & Urmia-Barouj & 1575.41 & 1883.44 & 3355.65 & 14 & 24 \\
\hline 4 & Urmia-Maranghalou & 1170.91 & 1615.43 & 3001.82 & 35.5 & 38 \\
\hline 5 & Marand-Dizaj- Ghalami & 2065.43 & 1917.77 & 3055.23 & 4.5 & 27 \\
\hline 6 & Jabalkandi 2 & 1415.56 & 2085.56 & 2695.38 & 31 & 31 \\
\hline 7 & Salmas - Sadaghian & 2217.95 & 2211.75 & 3177.21 & 3 & 19.5 \\
\hline 8 & Babaghanje 6 & 1002.17 & 2048.18 & 3505.74 & 35.5 & 17.5 \\
\hline 9 & Miyaneh-Basin & 1462.42 & 1273.47 & 1221.97 & 48 & 54 \\
\hline 10 & Boucan & 769.14 & 973.88 & 2103.75 & 50 & 51 \\
\hline 11 & Urmia - Nuoshinshahr & 1783.14 & 2482.86 & 3012.03 & 12.5 & 15.5 \\
\hline 12 & Karimabad & 1982.66 & 3611.45 & 3728.20 & 2 & 1 \\
\hline 13 & Vaghaslou-Olya 1 & 1439.29 & 1328.74 & 2768.42 & 28.5 & 42 \\
\hline 14 & Vaghaslou-Olya 3 & 1453.18 & 2686.43 & 3500.27 & 19 & 9 \\
\hline 15 & Ordoshahi 1 & 1611.69 & 2208.24 & 2852.48 & 23 & 25 \\
\hline 16 & Marana-Yamchi-Pesteii & 1434.58 & 2889.97 & 2802.96 & 28.5 & 13.5 \\
\hline 17 & Mazandaran-Tirtash & 996.16 & 1832.44 & 1675.26 & 49 & 43 \\
\hline 18 & Sardasht & 1500.16 & 1816.67 & 2356.11 & 35.5 & 41 \\
\hline 19 & Marana-Yamchi 4 & 1177.18 & 1299.26 & 1783.27 & 45.5 & 48 \\
\hline 20 & Salmas 2 & 965.81 & 2367.56 & 3037.67 & 42 & 17.5 \\
\hline 21 & Vaghaslou-Olya 4 & 1693.70 & 1927.74 & 2935.50 & 20 & 29.5 \\
\hline 22 & Salmas-Gharaghashlagh- Pesteii & 1901.66 & 2371.90 & 2691.73 & 17 & 23 \\
\hline 23 & Lalalou-Torab 2 & 1056.05 & 2002.13 & 3418.40 & 33 & 21 \\
\hline 24 & Shirabad 2 & 1588.41 & 2026.19 & 2591.75 & 26.5 & 33.5 \\
\hline
\end{tabular}




\begin{tabular}{|c|c|c|c|c|c|c|}
\hline 25 & Gharagoz 1 & 1746.63 & 3778.83 & 3528.58 & 6 & 2 \\
\hline 26 & Vaghaslou-Sofla 1 & 1471.15 & 2965.42 & 3635.39 & 12.5 & 5 \\
\hline 27 & Khanneshan 1 & 1703.78 & 2467.21 & 3037.68 & 15 & 15.5 \\
\hline 28 & Heydarlou 1 & 981.17 & 2908.40 & 3103.55 & 40.5 & 11 \\
\hline 29 & Saribaglou 5 & 2030.66 & 2853.26 & 3105.27 & 4.5 & 12 \\
\hline 30 & Chongharalou-Yekan 4 & 1567.05 & 2344.98 & 2243.27 & 35.5 & 33.5 \\
\hline 31 & Maranghalou 6 & 1985.41 & 3146.80 & 2961.98 & 8 & 10 \\
\hline 32 & Abajalou 1 & 1115.13 & 3596.70 & 3505.17 & 30 & 3 \\
\hline 33 & Hamadan 1 & 1453.90 & 3033.53 & 3513.70 & 17 & 6.5 \\
\hline 34 & Saghez 2 & 1807.28 & 1799.07 & 2523.31 & 25 & 39.5 \\
\hline 35 & Piranshahr-Serokani & 860.42 & 1439.76 & 1529.26 & 52 & 50 \\
\hline 36 & Piranshahr Andizeh & 497.26 & 493.39 & 1222.34 & 55 & 55 \\
\hline 37 & Mashhad & 490.16 & 515.86 & 807.34 & 56 & 56 \\
\hline 38 & Shahroud 1 & 1242.51 & 1315.93 & 1700.66 & 45.5 & 49 \\
\hline 39 & Hamadan 2 & 1217.73 & 1285.08 & 2071.94 & 43.5 & 46.5 \\
\hline 40 & Shabestar-Kouzeh-Kanan 3 & 689.73 & 1324.07 & 1426.11 & 53 & 52 \\
\hline 41 & Saghez 4 & 525.13 & 1177.20 & 1458.54 & 54 & 53 \\
\hline 42 & Saghez 5 & 1801.78 & 1645.39 & 3036.84 & 11 & 37 \\
\hline 43 & Saghez 3 & 2143.35 & 2183.06 & 2381.37 & 17 & 35.5 \\
\hline 44 & Shahroud 2 & 1062.24 & 1502.04 & 1942.53 & 47 & 44 \\
\hline 45 & Alibaglou 1 & 1396.10 & 1948.10 & 2331.95 & 38.5 & 39.5 \\
\hline 46 & Baneh 2 & 1727.82 & 2009.26 & 3241.56 & 10 & 22 \\
\hline 47 & $\begin{array}{c}\text { Salmas-Gharagheshlagh- } \\
\text { Ghalami }\end{array}$ & 1134.32 & 1987.24 & 4076.50 & 23 & 13.5 \\
\hline 48 & Marand-1389-2 & 1377.34 & 1721.08 & 3356.18 & 23 & 28 \\
\hline 49 & $\begin{array}{c}\text { Salmas-Gharaghashlagh- } \\
\text { Badami }\end{array}$ & 1788.36 & 2949.06 & 3340.35 & 7 & 8 \\
\hline 50 & Shabestar-Kouzeh Kanan 1 & 1370.58 & 2305.50 & 2390.03 & 38.5 & 32 \\
\hline 51 & Sanandaj & 1335.47 & 2045.97 & 2756.99 & 32 & 29.5 \\
\hline 52 & Shabestar-Kouzeh-Kanan 2 & 1222.83 & 1403.79 & 2030.41 & 43.5 & 45 \\
\hline 53 & Baneh 3 & 1153.55 & 1979.44 & 2624.47 & 40.5 & 35.5 \\
\hline 54 & Piranshahr-Baleban & 828.05 & 1522.93 & 1759.33 & 51 & 46.5 \\
\hline 55 & Baneh 1 & 1844.57 & 1902.77 & 3141.23 & 9 & 26 \\
\hline 56 & Marand-1389-1 & 1489.70 & 3228.56 & 3307.31 & 21 & 6.5 \\
\hline
\end{tabular}

\section{References}

Ahmadi G, Zeinaly Khane Ghah H, Rostamy MA, Chogan R (2000). The study of drought tolerance and biplot method in eight corn hybrids. Iranian Journal of Agriculture Science 31:513-523.

Ajalli J, Salehi M (2012). Evaluation of drought stress indices in barley (Hordeum vulgare L.). Annals of Biological Research 3 (12):5515-5520.

Ali Q, Ashraf M, Anwar F (2009). Physico-chemical attributes of seed oil from drought stressed sunflower (Helianthus annuus L.) plants. Grasas y Aceites 60:475-481.

Alizadeh A (2009). Soil and plant water relations. Press of Astan Ghods Razavi $484 \mathrm{p}$.

Anwar J, GHULAM Mahboob Subhani Genotype, Makhdoom H, Javed A, Mujahid H, Munir M (2011). Drought tolerance indices and their correlation with yield in exotic wheat genotypes. Pakistan J Bot 43(3):1527-1530.

Blum A (1988). Plant's breeding for Stress environments. CRC Press, Florida. P- 212.

Bouslama M, Schapaugh WT (1984). Stress tolerance in soybean. Part 1: evaluation of three screening techniques for heat and drought tolerance. Crop Sci 24:933-937.
Daneshian J, Jonoubi P (2008). Evaluation of sunflower new hybrids tolerance to water deficit stress. In proceeding of the 5th International Crop Science Congress. Jejo, Korea. Pp:189.

Dehbalaei S, Farshadfar E, Farshadfar M (2013). Assessment of drought tolerance in bread wheat genotypes based on resistance/ tolerance indices. International Journal of Agriculture and Crop Sciences 5(20):2352-2358.

Dehghani GH, Malekshhi F, Alizadeh B (2009). A study of drought tolerance indices in canola (Brassica napus L.) genotypes. Journal of Science and Technology of Agriculture and Natural Resources 13(48):77-90.

Drikvand R, Doosty B, Hosseinpour T (2012). Response of rainfed wheat genotypes to drought stress using drought tolerance indices. J Agr Sci 4(7):126-131.

Ebrahimzadeh Banayjedi M, Ehteshami SM, Delkhosh H, Abdi B, Rohami M (2012). Evaluation of drought tolerance indices and their relationship with grain yield of wheat genotypes. $\mathrm{J}$ Basic Appl Sci Res 2(2):1230-1232.

Eivazi AR, Mohammadi S, Rezaei M, Ashori S, Hossien Pour F (2013). Effective selection criteria for assessing drought tolerance indices in barley (Hordeum vulgare L.) accessions. International Journal of Agronomy and Plant Production 4 (4):813-821. 
200

Farshadfar E, Ghannadha M, Zahravi M, Sutka J (2001). Genetic analysis of drought tolerance in wheat. Plant Breeding 114:542-544.

Farshadfar E, Moradi Z, Elyasi P, Jamshidi B, Chaghakabodi R (2012). Effective selection criteria for screening drought tolerant landraces of bread wheat (Triticum aestivum L.). Annals of Biological Research 3(5):2507-2516.

Farshadfar E, Poursiahbidi MM, Safavi SM (2013). Assessment of drought tolerance in land races of bread wheat based on resistance/tolerance indices. International Journal of Advanced Biological and Biomedical Research 1(2):143-158.

Farshadfar E, Sutka J (2002). Screening drought tolerance criteria in maize. Acta Agronomica Hungarica 50(4):411-416.

Fernandez GCJ (1992). Effective selection criteria for assessing stress tolerance. In: Kuo C.G. (Ed.), Proceedings of the International Symposium on Adaptation of Vegetables and Other Food Crops in Temperature and Water Stress, Publication, Tainan, Taiwan.

Fischer RA, Maurer R (1978). Drought resistance in spring wheat cultivars. I. Grain yield response. Aust J Agric Res 29:897-912.

Gabriel KR (1971). The biplot graphic display of matrics with application to principal component analysis. Biometrika 58:453-467.

Ganjali A, Bagheri A, Porsa H (2009). Evaluation of chickpea (Cicer arietinum $\mathrm{L}$.) germplasm for drought resistance. Journal of Agronomic Research of Iran 7(1):183-194.

Gavuzzi P, Rizza F, Palumbo M, Campaline RG, Ricciardi GL, Borghi B (1997). Evaluation of field and laboratory predictors of drought and heat tolerance in winter cereals. Can J Plant Sci 77:523-531.

Golabadi M, Arzani A, Maibody SAM (2006). Assessment of drought tolerance in segregating populations in durum wheat. African Journal of Agriculture Research 5:162-171.

Ilker E, Tatar O, Aykut Tonk F, Tosun M, Turk J (2011). Determination of Tolerance Level of Some Wheat Genotypes to Post-Anthesis Drought. Turkish Journal of Field Crops 16(1):59-63.

Jabbari H, Akbari GA, Daneshian J, Alahdadi I, Shahbazian N (2008). Utilization ability of drought resistance indices in sunflower (Heliantus annus L.) hybrids. EJCP 1(4):1-17.

Jafari A, Paknejad F, Al-Ahmadi M (2009). Evaluation of selection indices for drought tolerance of corn (Zea mays L.) hybrids. Int J Plant Product 3:33-38.

Jaleel CA, Manivannan P, Sankar B, Kishorekumar A, Gopi R, Somasundaram R, Panneerselvam R (2007). Induction of drought stress tolerance by Ketoconazole in Catharanthus roseus is mediated by enhanced antioxidant potentials and secondary metabolite accumulation. Colloids Surf B Biointerfaces 60:201- 206.

Kaya Y, Plta C, Taner S (2002). Additive main effects and multiplicative interaction analysis of yield performance in bread wheat genotypes across environments. Turk J Agric 26:257-259.
Khalili M, Naghavi MR, Pour Aboughadareh AR, Talebzadeh SJ (2012). Evaluating of drought stress tolerance based on selection indices in spring canola cultivars (Brassica napus L.). Journal of Agricultural Science 4(11):78-85.

Khodarahmpour Z, Choukan R, Bihamta MR, Majidi Hervan E (2011). Determination of the best heat stress tolerance indices in maize (Zea mays L.) inbred lines and hybrids under Khuzestan province conditions. Iranian Journal of Crop Sciences 13:111-121.

Kristin AS, Serna RR, Perez FI, Enriquez BC, Gallegos JAA, Vallejo PR, Wassimi N, Kelly JD (1997). Improving common bean performance under drought stress. Crop Sci 37:51-60.

Lan J (1998). Comparison of evaluating methods for agronomic drought resistance in crops. Acta Agric Boreali-occidentalis Sinica 7:85-87.

Lu G, Hoeft E (2007). Sunflower. In Kole C, Hall TC (eds.), A compendium of transgenic crop plants Vol. 2, Oxford, WileyBlackwell.

Mevlut A, Sait C (2011). Evaluation of drought tolerance indices for selection of Turkish oat (Avena sativa L.) landraces under various environmental conditions. Zemdirbyste Agriculture 98(2):157-166.

Mitra J (2001). Genetics and genetic improvement of drought resistance in crop plants. Curr Sci India 80:758-762.

Moghadam A, Hadizadeh MH (2000). Study in density stress in selection of drought tolerant varieties in corn. Iranian Journal of Crop Sci 2(3):25-38.

Mohammadi R, Armion M, Kahrizi D, Amri A (2010). Efficiency of screening techniques for evaluating durum wheat genotypes under mild drought conditions. Int J Plant Product 4(1):1735-8043.

Moosavi SS, Yazdi Samadi B, Naghavi MR, Zali AA, Dashtid H, Pourshahbazi A (2008). Introduction of new indices to identify relative drought tolerance and resistance in wheat genotypes. Desert 12:165-178.

Pourdad SS, Alizadeh KH, Azizinezhad R, Shariati A, Askandari M, Khiyavi M, Ezatollahe N (2008). Evaluation of different safflowers in different regions. Sci Tech Agri Natur Resources 12 (45):403-415.

Razi H, Assad MT (1999). Comparison of seletion criteria in normal and limited irrigation in sunflower. Euphytica 105: 8390.

Rosielle AA, Hamblin J (1981). Theoretical aspects of selection for yield in stress and non-stress environment. Crop Sci 21:943-946.

Safahani Langeroodi AR, Kamkar B, Ataei M, Teixeira Da Silva A (2013). Assessment of the response of sunflower cultivars to water shortage using various stress tolerance indices. Int $\mathrm{J}$ Agron Plant Prod 4 (7):1628-1636.

Sajad Bokaei A, Babaei H, Habibi D, Javidfar S, Mohammadi A (2008). Evaluation of different soybean (Glycine max L.) genotypes under drought stress conditions. Journal of Agronomy and Plant Breeding 4(1):28-38. 
Salunkhe DK, Chavan JK, Adsule RN, Kadam S S (1999). World oil seeds: Chemistry, technology, and utilization. New York: Van Nostrand Reinhold.

Sio-Se Mardeh A, Ahmadi A, Poustini K, Mohammadi V (2006). Evaluation of drought resistance indices under various environmental conditions. Field Crop Res 98:222-229.

Tabatabaei SA (2013). Study relationship of drought tolerance indices in wheat (Triticum aestivum) genotypes. International Journal of Biosciences 3(7):15-22.

Tollenaar M, Wu J (1999). Yield improvement in temperate maize is attributable to greater stress tolerance. Crop Sci 39:1597-1604.

Toorchi M, Naderi R, Kanbar A, Shakiba MR (2012). Response of spring canola cultivars to sodium chloride stress. Annals of Biological Research 2(5):312-322.
Zaheri A, Bahraminejad Sunflower (2012). Assessment of drought tolerance in oat (Avena sativa) genotypes. Annals of Biological Research 3(5):2194-2201.

Zahravi M (2009). Evaluation of genotypes of wild barley (Hordeum spontaneum) based on drought tolerance indices. Seed and Plant 25(4):533-549.

Zare M (2012). Evaluation of drought tolerance indices for the selection of Iranian barley (Hordeum vulgare) cultivars. African Journal of Biotechnology 11(93):15975-15981. 